Recepción: 20 / 04 / 2017

Aceptación: 20 / 05 / 2017

Publicación: 15 / 06 / 2017

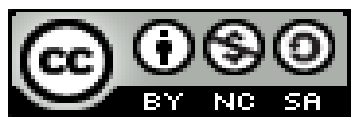

Ciencias de la Educación

Artículo de Investigación

\title{
La contribución del uso de la web 2.0 al nivel de competencias tecnológicas
}

\author{
The contribution of the use of the Web 2.0 to the level of \\ technological competences
}

\section{A contribuição do uso da Web 2.0 com o nível de competências tecnológicas}

Renzo R. Padilla-Gómez ${ }^{\text {I }}$ renzo.padillag@ug.edu.ec

Silvia M. Chicaiza-Guayta ${ }^{\text {II }}$ schicaiza@uagraria.edu.ec

María M. Amaguaya-Vargas ${ }^{\text {III }}$ mamaguaya@uagraria.edu.ec

Correspondencia: renzo.padillag@ug.edu.ec

Ingeniero en Sistemas Computacionales, Magister en Educación Informática, Magister en Docencia y Gerencia en Educación Superior, Universidad de Guayaquil, Guayaquil, Ecuador.

II. Analista de Sistemas, Ingeniera en Sistemas Computacionales, Magister en Educación Informática, Universidad Agraria del Ecuador, Guayaquil, Ecuador.

III. Magister en Educación Informática; Magister en Docencia y Gerencia en Educación Superior; Ingeniera en Sistemas Computacionales; Analista de Sistemas; Universidad Agraria del Ecuador, Guayaquil, Ecuador. 


\section{Resumen}

Actualmente la sociedad se encuentra sumergida en un proceso de cambio promovido por los avances en las tecnologías de la información y comunicación. El sistema educativo universitario debe estar capacitado para hacer frente a estos avances tecnológicos, siendo la formación del docente el elemento clave para facilitar la integración de los planes analíticos, tal y como se demanda desde diversos entornos sociales, institucionales, entre otros. La formación del docente debe ir encaminada a innovar, experimentar con las TICS y reflexionar sobre su utilidad, beneficiando el desarrollo y la adquisición de distintas competencias tecnológicas que le capaciten para usar de forma didáctica las herramientas de la web 2.0, convirtiéndose en un recurso más dentro del aula de clases. El problema planteado guarda relación con la siguiente interrogante de la investigación ¿Cómo contribuye el uso de la web 2.0 al nivel de competencias tecnológicas de los docentes del Sistema Nacional de Nivelación de la Universidad Estatal de Milagro en el año 2014?. Este trabajo tiene su fundamentación teórica que abarca todas las teorías investigaciones que hablan sobre las TICS en la educación con una base Filosófica, Psicológica, Sociológica, Filosófica y Legal. Es un proyecto factible donde se utilizaron las técnicas de entrevista y encuestas que se aplicaran a la muestra de la población que la representó por coordinadores, docentes y estudiantes. La información que se recogió fue representada en cuadros estadísticos y en gráficos, lo que permitió realizar la discusión de los resultados poniendo en marcha la propuesta que radica en el diseño de un manual académico tecnológico para docentes.

Palabras claves: Competencias tecnológicas; Manual académico; Web 2.0. 


\begin{abstract}
At the moment the society is immersed in a process of change promoted by the advances in the technologies of the information and communication. The university education system must be able to cope with these technological advances, with teacher training being the key element to facilitate the integration of analytical plans, as demanded from various social and institutional environments, among others. The training of teachers should be aimed at innovating, experimenting with ICTs and reflecting on their usefulness, benefiting the development and acquisition of different technological competences that enable them to use didactically the tools of Web 2.0, becoming a more resource Inside the classroom. The problem raised is related to the following question of the research How does the use of web 2.0 contribute to the level of technological competences of the teachers of the National Leveling System of the State University of Miracle in 2014? This work has its theoretical foundation that covers all the research theories that talk about ICTs in education with a Philosophical, Psychological, Sociological, Philosophical and Legal basis. It is a feasible project where interview techniques and surveys were used that would be applied to the sample of the population that represented it by coordinators, teachers and students. The information collected was represented in statistical tables and graphs, which allowed the discussion of the results to be carried out, putting in motion the proposal that lies in the design of an academic academic manual for teachers.
\end{abstract}

Key words: Technological competences; Academic Handbook; Web 2.0. 


\section{Resumo}

Atualmente a empresa está imerso em um processo de mudança promovido pelos avanços na tecnologia da informação e comunicação. O sistema de ensino universitário deve ser capaz de lidar com esses avanços tecnológicos, a formação de professores a ser a chave para facilitar a integração dos planos de análise, como a demanda de vários, ambientes institucionais sociais, entre outros elementos. formação de professores devem procurar inovar, experimentar com TICS e refletir sobre a sua utilidade, beneficiando o desenvolvimento e aquisição de várias habilidades tecnológicas que lhe permitem utilizar didática ferramentas da Web 2.0, tornando-se um recurso dentro da sala de aula. O problema levantado diz respeito à seguinte questão de pesquisa Como é que o uso da tecnologia web 2.0 para o nível de competências dos professores do Sistema Nacional de Equalização Milagro State University em 2014?. Este trabalho tem o seu fundamento teórico que abrange todas as teorias de pesquisa falar sobre TIC na educação com uma base filosófica, psicológica, sociológica, filosófica e jurídica. É um projeto viável, onde técnicas de entrevista e questionários foram aplicados à amostra da população representada pelos coordenadores, professores e alunos foram utilizados. As informações coletadas foram representadas em quadros estatísticos e gráficos, que permitiu a discussão dos resultados através da implementação da proposta reside na concepção de um manual acadêmico tecnológico para os professores.

Palavras-chave: competências tecnológicas; Manual do acadêmico; Web 2.0. 


\section{Introducción.}

Actualmente la educación a nivel mundial se enfrenta el desafío del uso de la tecnología como herramienta para el proceso de enseñanza aprendizaje con el propósito de que el educando desarrolle destrezas que le va a servir para enfrentar desafíos y dar solución a las necesidades de la sociedad futura.

La web 2.0 deja a un lado la enseñanza tradicional donde los estudiantes solo eran receptores de la información que emitía el docente, sino que esta herramienta permite la interacción permitiendo la retroalimentación de información entre estudiantes y docentes.

Estas herramientas que encontramos en internet de forma gratuita ayudan a desarrollar actividades académicas innovadoras dejando a un lado los medios cotidianos como videos de la televisión, revistas, periódicos, entre otros. La tecnología que tiene la Web 2.0 es fácil de usar que hasta los usuarios con poco conocimiento tecnológico podrán hacer uso de estas herramientas.

Hay que tomar en cuenta que se debe de reconstruir el entorno educativo donde implica la adaptación de nuevas estrategias de enseñanza aprendizaje donde se involucre este tipo de herramientas para que ayude a incentivar al estudiante logrando captar su interés que beneficie su aprendizaje.

Como parte final de la investigación se presentan las conclusiones y recomendaciones, las cuales reflejarán el cumplimiento de los objetivos, resultados principales, aceptación o rechazo de la hipótesis, adjunto la bibliografía y los anexos de la investigación.

\section{Materiales y métodos.}

Los métodos utilizados en esta investigación son:

Hipotético Deductivo.- Se realizó un estudio que se basó en la comprobación de la hipótesis y que este nos lleve al estudio de la contribución en el uso de la web 2.0 al nivel de competencias tecnológicas con el propósito de utilizar de la manera más óptima estas herramientas para el proceso de enseñanza aprendizaje de los estudiantes.

Analítico Sintético.- Se reconoció el problema existente en el curso de nivelación y admisión respecto de cómo contribuye el uso de la web 2.0 al nivel de competencias tecnológicas de los 
docentes, para ello hay que descomponer las partes o elementos que involucran esta problemática y poder entender a cada elemento y cada proceso, para alcanzar un equilibrio y así cumplir los objetivos planteados

\section{Resultados.}

Encuestas dirigidas a los docentes del curso de nivelación y admisión de la "Universidad Estatal de Milagro"

\begin{tabular}{lcc}
\hline \multicolumn{1}{c}{ Alternativas } & Cantidad & \% \\
\hline Hogar & 44 & $95,65 \%$ \\
Universidad & 46 & $100,00 \%$ \\
Cyber & 2 & $4,35 \%$ \\
Otros & 19 & $41,30 \%$ \\
Total & & \\
\hline
\end{tabular}

\section{Pregunta 1: ¿Tiene acceso a internet en?}

Cuadro 1: Frecuencia de si se tiene acceso al internet

Fuente: Encuesta realizada a los docentes del curso de nivelación SNNA-UNEMI

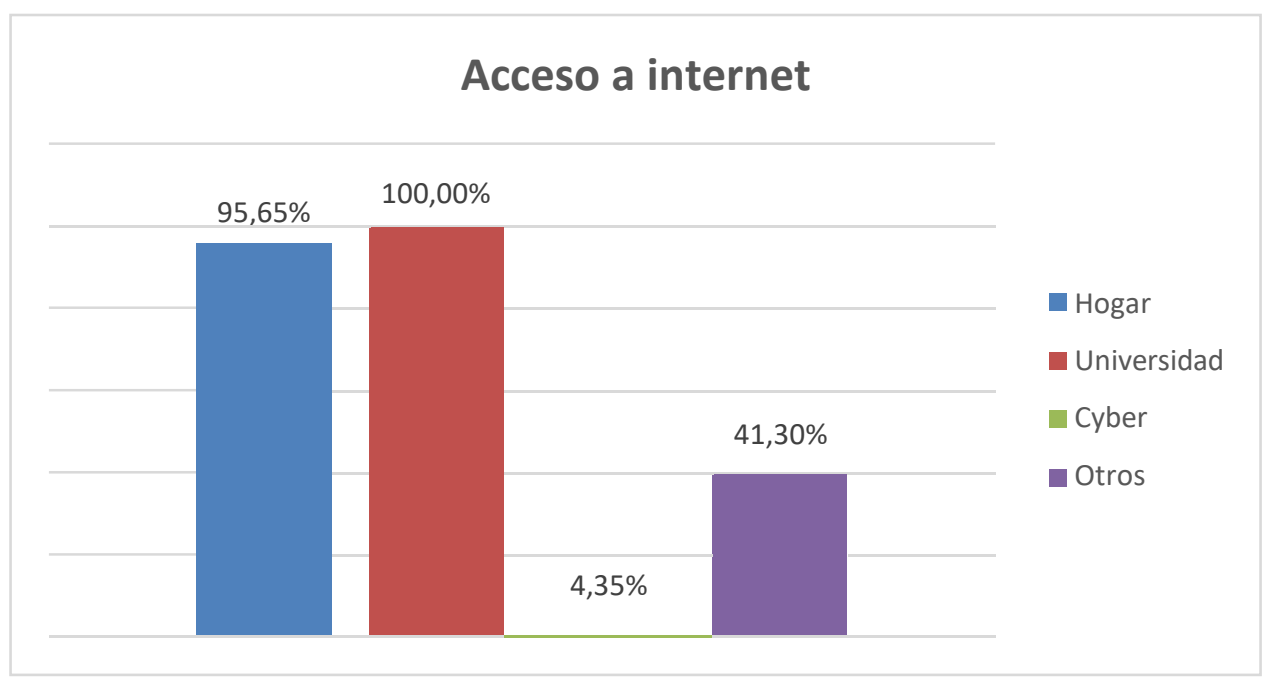

Gráfico 1: Frecuencia de si se tiene acceso al internet

Fuente: Encuesta realizada a los docentes del curso de nivelación SNNA-UNEMI

\section{Análisis}

El 95,65\% de los docentes indican que tienen acceso a internet en el hogar, el 100\% en la universidad, el 4,35\% en el cyber y el $41,30 \%$ otros (celular, parques, mall, entre otros).

En los resultados obtenidos por los docentes se puede apreciar que todos tienen acceso a internet en la universidad y la gran mayoría fuera de ésta. 


\begin{tabular}{lcc}
\hline Todos los días & 42 & $91,30 \%$ \\
Varios días a la semana & 3 & $6,52 \%$ \\
Una vez a la semana & 1 & $2,17 \%$ \\
Nunca & 0 & $0,00 \%$ \\
Total & 46 & $100,00 \%$ \\
\hline
\end{tabular}

\section{Pregunta2: ¿Con que frecuencia acceso a internet:?}

Cuadro 2: Frecuencia de acceso al internet

Fuente: Encuesta realizada a los docentes del curso de nivelación SNNA-UNEMI

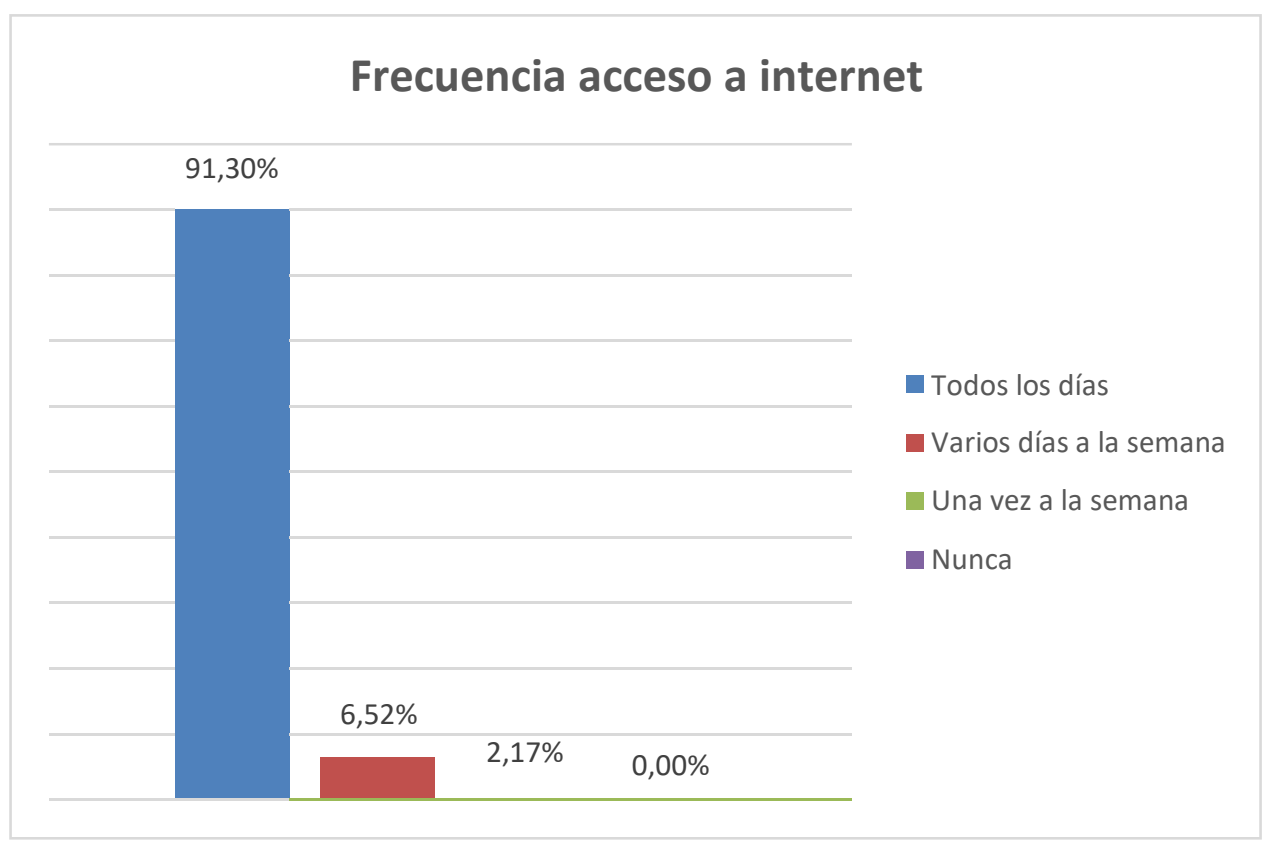

Gráfico 2: Frecuencia de acceso al internet

Fuente: Encuesta realizada a los docentes del curso de nivelación SNNA-UNEMI

\section{Análisis}

El 91,30\% de los docentes encuestados mencionan que todos los días accedan a internet, el $6,52 \%$ varios días a la semana, el 2,17\% una vez a la semana y el $0 \%$ nunca.

En los resultados obtenidos por los docentes se puede apreciar que todos accedan a internet la mayor parte del tiempo.

\begin{tabular}{lcc}
\hline Alternativas & Cantidad & \% \\
\hline Compartir información & 7 & $15,22 \%$ \\
\hline
\end{tabular}




\begin{tabular}{lcr}
\hline Comunicación (email,facebook,etc) & 18 & $39,13 \%$ \\
Entretenimiento & 15 & $32,61 \%$ \\
Otros & 6 & $13,04 \%$ \\
Total & 46 & $100,00 \%$ \\
\hline
\end{tabular}

Pregunta 3: ¿Cuál de las siguientes actividades realiza con frecuencia en el internet?

Cuadro 3: Frecuencia de actividades en el internet

Fuente: Encuesta realizada a los docentes del curso de nivelación SNNA-UNEMI

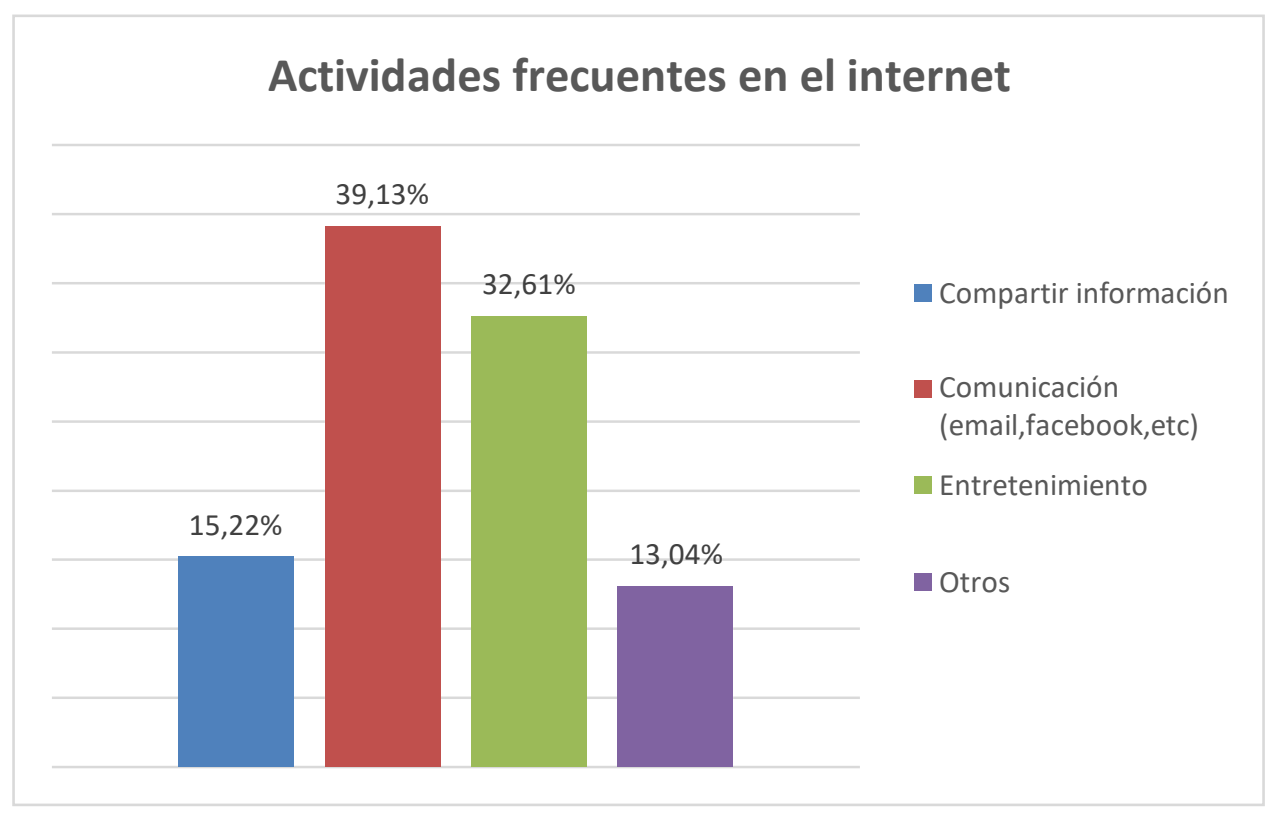

Gráfico 3: Frecuencia de actividades en el internet

Fuente: Encuesta realizada a los docentes del curso de nivelación SNNA-UNEMI

\section{Análisis}

De los docentes encuestados sobre las actividades que realizan con frecuencia en el internet, el 15,22\% indican que comparten información, el 39,13\% lo usan como medio de comunicación, el $32,61 \%$ para entretenimiento, el 13,04\% otros.

En los resultados obtenidos por los docentes se puede apreciar que la tendencia es la comunicación y entretenimiento, es decir que lo usan para diversión. 
Renzo R. Padilla-Gómez; Silvia M. Chicaiza-Guayta; María M. Amaguaya-Vargas

\begin{tabular}{lcc}
\hline \multicolumn{1}{c}{ Alternativas } & Cantidad & \% \\
\hline Facebook & 38 & $82,61 \%$ \\
Google Drive & 23 & $50,00 \%$ \\
Slideshare & 14 & $30,43 \%$ \\
Youtube & 36 & $78,26 \%$ \\
Ninguna & 3 & $6,52 \%$ \\
Total & & \\
\hline
\end{tabular}

Pregunta 4: ¿Usted conoce las siguientes herramientas tecnológicas de la web 2.0?

Cuadro 4: Conocimiento de herramientas web 2.0

Fuente: Encuesta realizada a los docentes del curso de nivelación SNNA-UNEMI

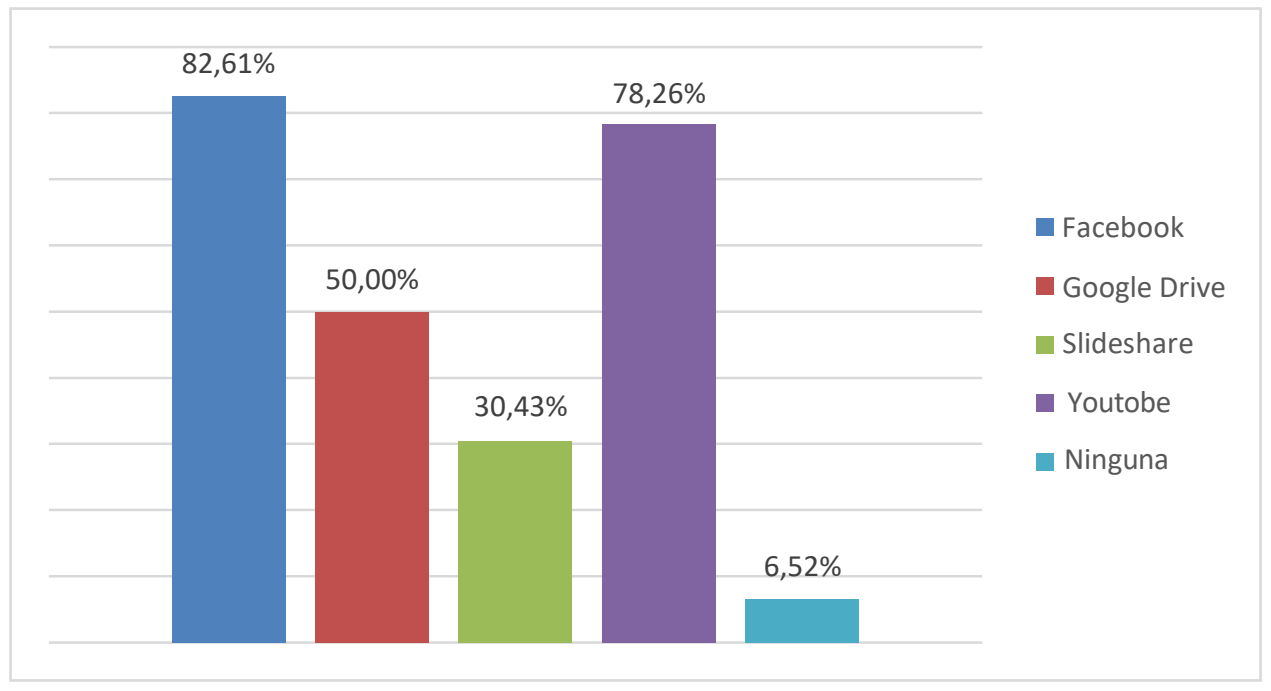

Gráfico 4: conocimiento de herramientas web 2.0

Fuente: encuesta realizada a los docentes del curso de nivelación SNNA-UNEMI

\section{Análisis}

De los docentes encuestados sobre las herramientas tecnológicas de la web 2.0 que conoce el 82,61\% indican que el Facebook, el 50\% Google Drive, el 30,43\% Slideshare, el 78,26\% Youtube, el $6,52 \%$ ninguna.

En los resultados obtenidos por los docentes se puede apreciar que la mayor parte conoce ciertas herramientas pero desconocen su uso en el área educativa. 


\begin{tabular}{lcc}
\hline \multicolumn{1}{c}{ Alternativas } & Cantidad & \% \\
\hline Facebook & 1 & $2,17 \%$ \\
Google Drive & 3 & $6,52 \%$ \\
Slideshare & 1 & $2,17 \%$ \\
Youtube & 13 & $28,26 \%$ \\
Ninguna & 28 & $60,87 \%$ \\
Total & 46 & $100,00 \%$ \\
\hline
\end{tabular}

Pregunta 5: ¿Cuál de las siguientes herramientas Web 2?0. ¿Utiliza con frecuencia en el aula de clases?

Cuadro 5: Herramientas Web 2.0 que se usa en el aula Fuente: Encuesta realizada a los docentes del curso de nivelación SNNA-UNEMI

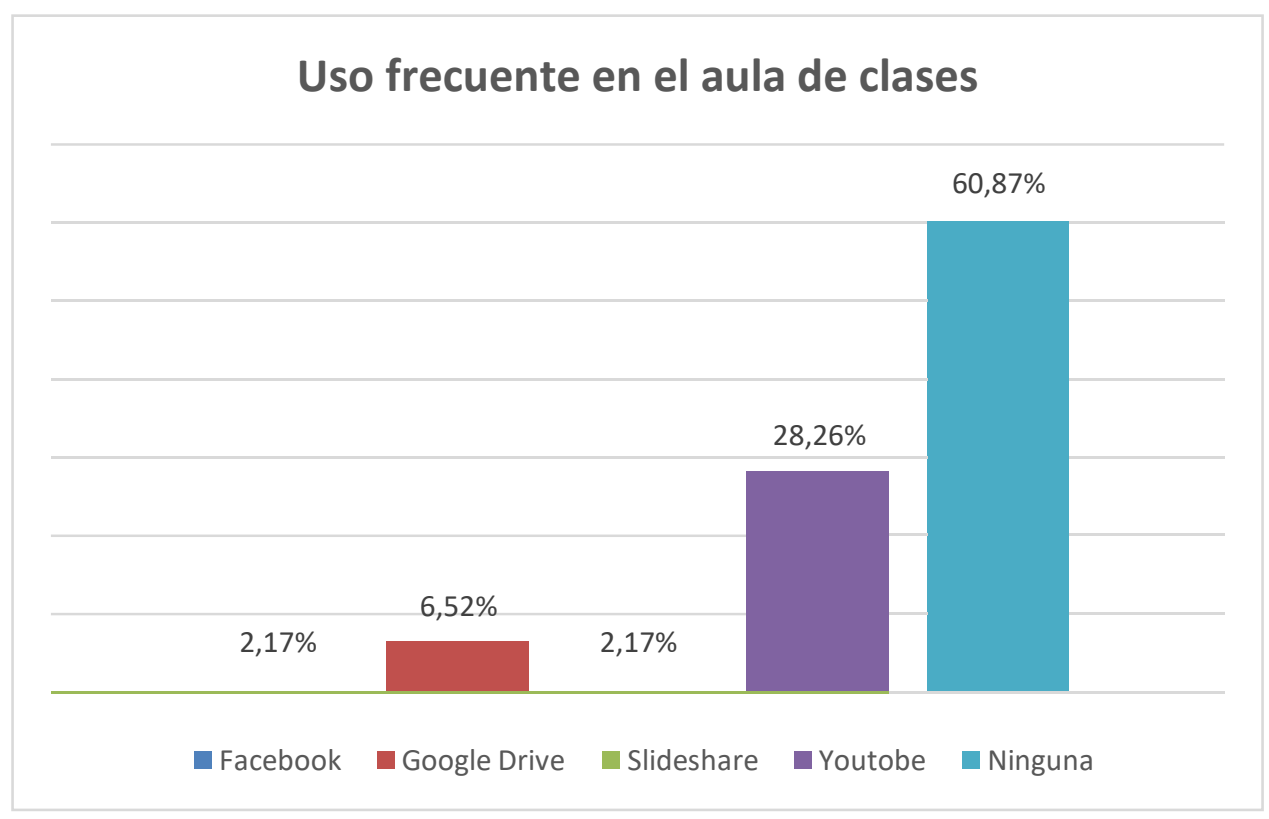

Gráfico 5: Herramientas Web 2.0 que se usa en el aula Fuente: Encuesta realizada a los docentes del curso de nivelación SNNA-UNEMI

\section{Análisis}

El 2,17\% de los docentes usa con frecuencia en el aula de clases el Facebook, el 6,52\% Google Drive, el 2,17\% slideshare, el 28,26\%, Youtube, el 60,87\% ninguna.

En los resultados obtenidos por los docentes se puede apreciar que la mayoría no usa las herramientas web 2.0 en el aula de clases. 
Renzo R. Padilla-Gómez; Silvia M. Chicaiza-Guayta; María M. Amaguaya-Vargas

\begin{tabular}{lcc}
\hline \multicolumn{1}{c}{ Alternativas } & Cantidad & \% \\
\hline Correo electrónico & 19 & $41,30 \%$ \\
Paginas Sociales & 7 & $15,22 \%$ \\
Aulas Virtuales & 46 & $100,00 \%$ \\
Otros & 0 & $0,00 \%$ \\
Ninguno & 21 & $45,65 \%$ \\
Total & & \\
\hline
\end{tabular}

Pregunta 6: ¿Utiliza alguna herramienta tecnológica para comunicarse con sus estudiantes? Cuadro 6: Uso de herramienta para comunicación Fuente: Encuesta realizada a los docentes del curso de nivelación SNNA-UNEMI

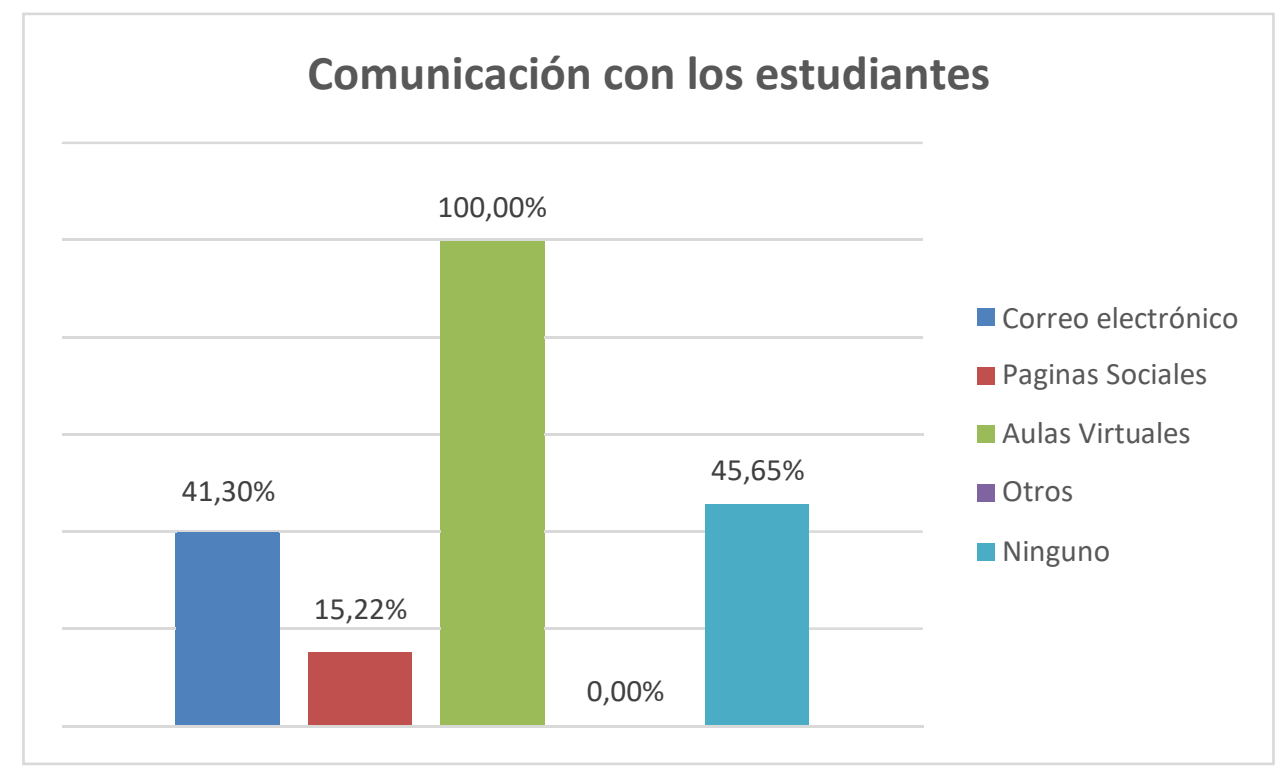

Gráfico 6: Uso de herramienta para comunicación

Fuente: Encuesta realizada a los docentes del curso de nivelación SNNA-UNEMI

Análisis

El 41,30\% usa el correo el correo electrónico para comunicarse con los estudiantes, el $15,22 \%$ paginas sociales, el $100 \%$ aulas virtuales, el $0 \%$ otros, el $45,65 \%$ ninguno.

El 100\% de los docentes usan las aulas virtuales porque les exige la Universidad, muy poco usan los otros medios. 


\begin{tabular}{lcc}
\hline \multicolumn{1}{c}{ Alternativas } & Cantidad & \% \\
\hline Educación & 10 & $21,74 \%$ \\
Conocer Personas & 19 & $41,30 \%$ \\
Entretenimiento & 15 & $32,61 \%$ \\
No utiliza & 2 & $4,35 \%$ \\
Total & 46 & $100,00 \%$ \\
\hline
\end{tabular}

Pregunta 7: ¿En qué ámbitos ha utilizado las herramientas Web 2.0 tales como facebook, Google Drive, Slideshare, Youtube?

Cuadro 71: Ámbitos en que utiliza la web 2.0

Fuente: Encuesta realizada a los docentes del curso de nivelación SNNA-UNEMI

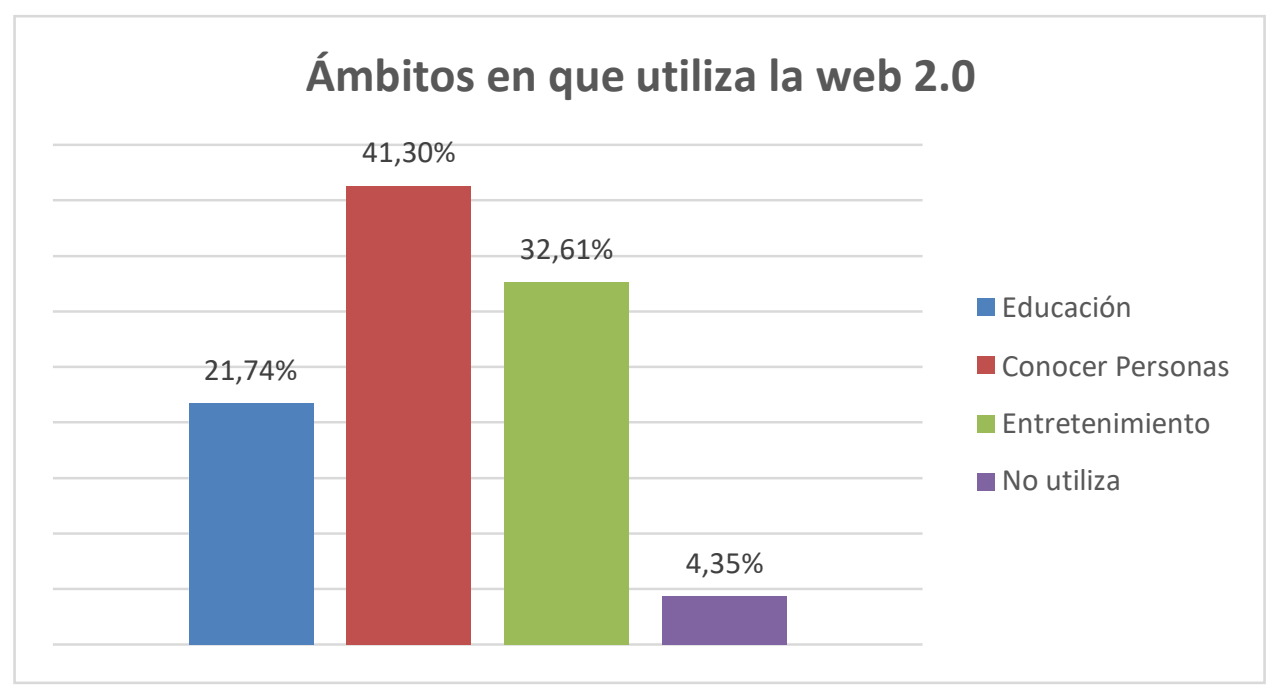

Gráfico 7: Ámbitos en que utiliza la web 2.0

Fuente: Encuesta realizada a los docentes del curso de nivelación SNNA-UNEMI

\section{Análisis}

El 21,74\% de los docentes usan las herramientas web 2.0 en el ámbito educativo, el 41,30\% para conocer personas, el 32,61\% para entretenimiento y el 4,35\% no utiliza.

En los resultados obtenidos por los docentes se puede apreciar que una menor parte lo usan para el ámbito educativo, más es usado para conocer personas y entretenimiento. 


\begin{tabular}{lcr}
\hline \multicolumn{1}{c}{ Alternativas } & Cantidad & \% \\
\hline Muy de acuerdo & 13 & $28,26 \%$ \\
De acuerdo & 26 & $56,52 \%$ \\
Indiferente & 5 & $10,87 \%$ \\
En desacuerdo & 2 & $4,35 \%$ \\
Total & 46 & $100,00 \%$ \\
\hline
\end{tabular}

Pregunta 8: ¿Considera que los medios de comunicación electrónicos son una herramienta que apoya al envio y recepción de tarea?

Cuadro 8: Medios de comunicación electrónicos

Fuente: Encuesta realizada a los docentes del curso de nivelación SNNA-UNEMI

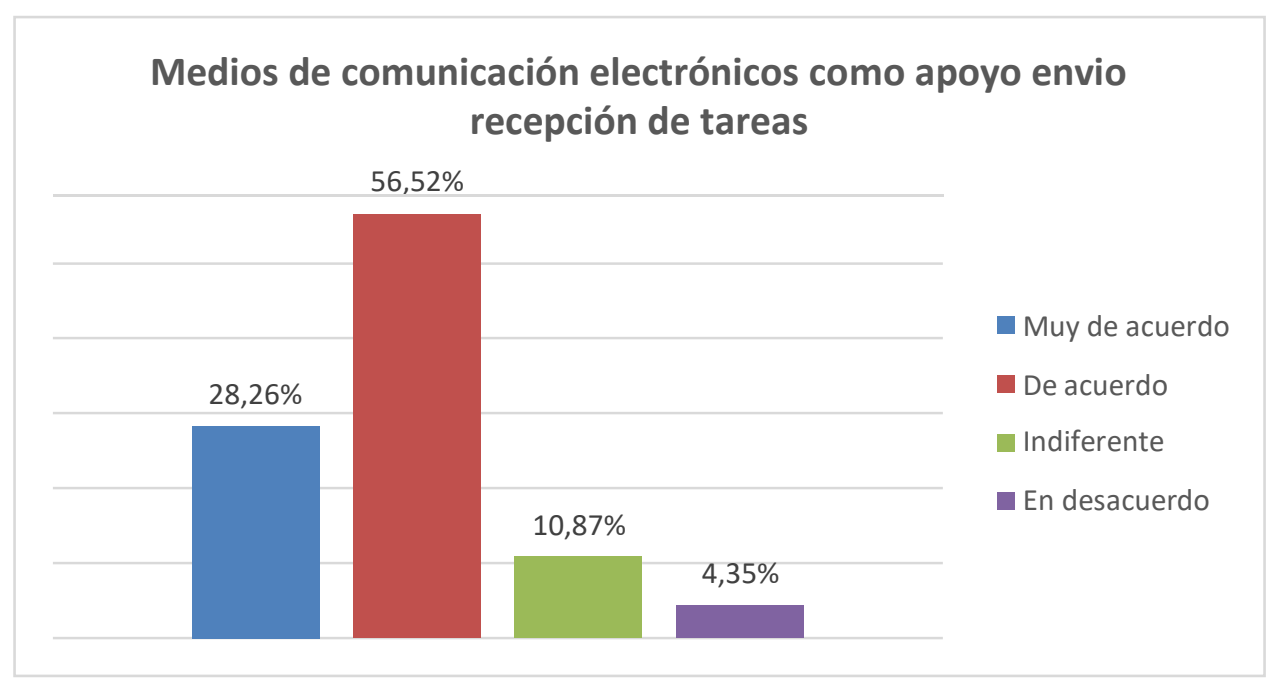

Gráfico 8: Medios de comunicación electrónicos

Fuente: Encuesta realizada a los docentes del curso de nivelación SNNA-UNEMI

\section{Análisis}

El 28,26\% de los docentes encuestados están muy de acuerdo que los medios de comunicación electrónicos son una herramienta que apoya al envío y recepción de tarea, el 56,52\% de acuerdo, el 10,87\% indiferente y el 4,35\% en desacuerdo.

En los resultados obtenidos por los docentes se puede apreciar que hay una gran aceptación que se utilice medios de comunicación electrónicos para presentación de tareas. 


\begin{tabular}{lcr}
\hline \multicolumn{1}{c}{ Alternativas } & Cantidad & \% \\
\hline Muy de acuerdo & 45 & $97,83 \%$ \\
De acuerdo & 1 & $2,17 \%$ \\
Indiferente & 0 & $0,00 \%$ \\
En desacuerdo & 0 & $0,00 \%$ \\
Total & 46 & $100,00 \%$ \\
\hline
\end{tabular}

Pregunta 9: ¿Es indispensable que los docentes conozca el uso educativo de la web 2.0 (herramientas tecnológicas que están en la internet), como mejoramiento al perfil de competencias tecnológicas?

Cuadro 9: Conocimiento sobre el uso educativo web 2.0

Fuente: Encuesta realizada a los docentes del curso de nivelación SNNA-UNEMI

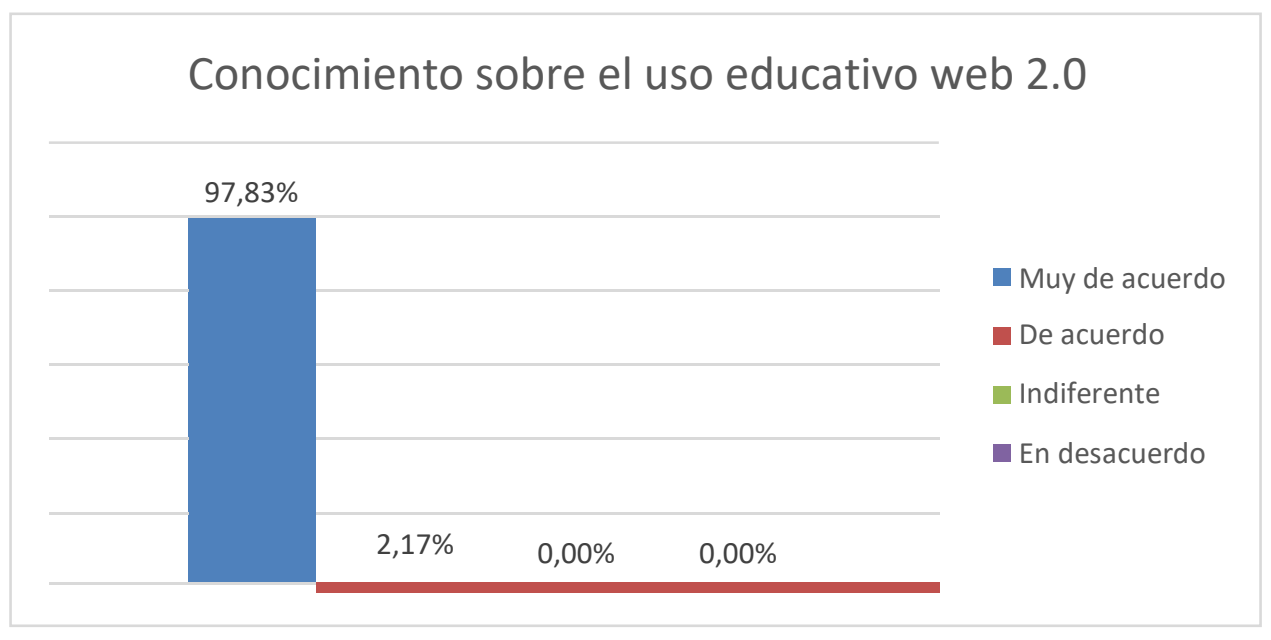

Gráfico 9: conocimiento sobre el uso educativo web 2.0

Fuente: encuesta realizada a los docentes del curso de nivelación SNNA-UNEMI

\section{Análisis}

El 19,57\% de los docentes encuestados están muy de acuerdo que se conozca el uso educativo de la web 2.0 como mejoramiento al perfil de competencias tecnológicas, el 76,09\% de acuerdo, el 2,17\% indiferente y el 2,17\% en desacuerdo.

En los resultados obtenidos por los docentes se puede apreciar que hay una gran aceptación en el uso de este tipo de herramientas para mejorar su perfil tecnológico y así estar a la vanguardia de la tecnología. 


\begin{tabular}{lcr}
\hline \multicolumn{1}{c}{ Alternativas } & Cantidad & \% \\
\hline Muy de acuerdo & 14 & $30,43 \%$ \\
De acuerdo & 17 & $36,96 \%$ \\
Indiferente & 10 & $21,74 \%$ \\
En desacuerdo & 5 & $10,87 \%$ \\
Total & 46 & $100,00 \%$ \\
\hline
\end{tabular}

Pregunta 10: ¿Considera Usted que la Web 2.0 beneficia la formación de los estudiantes?

Cuadro 10: Beneficio Web 2.0 en la formación de estudiantes

Fuente: Encuesta realizada a los docentes del curso de nivelación SNNA-UNEMI

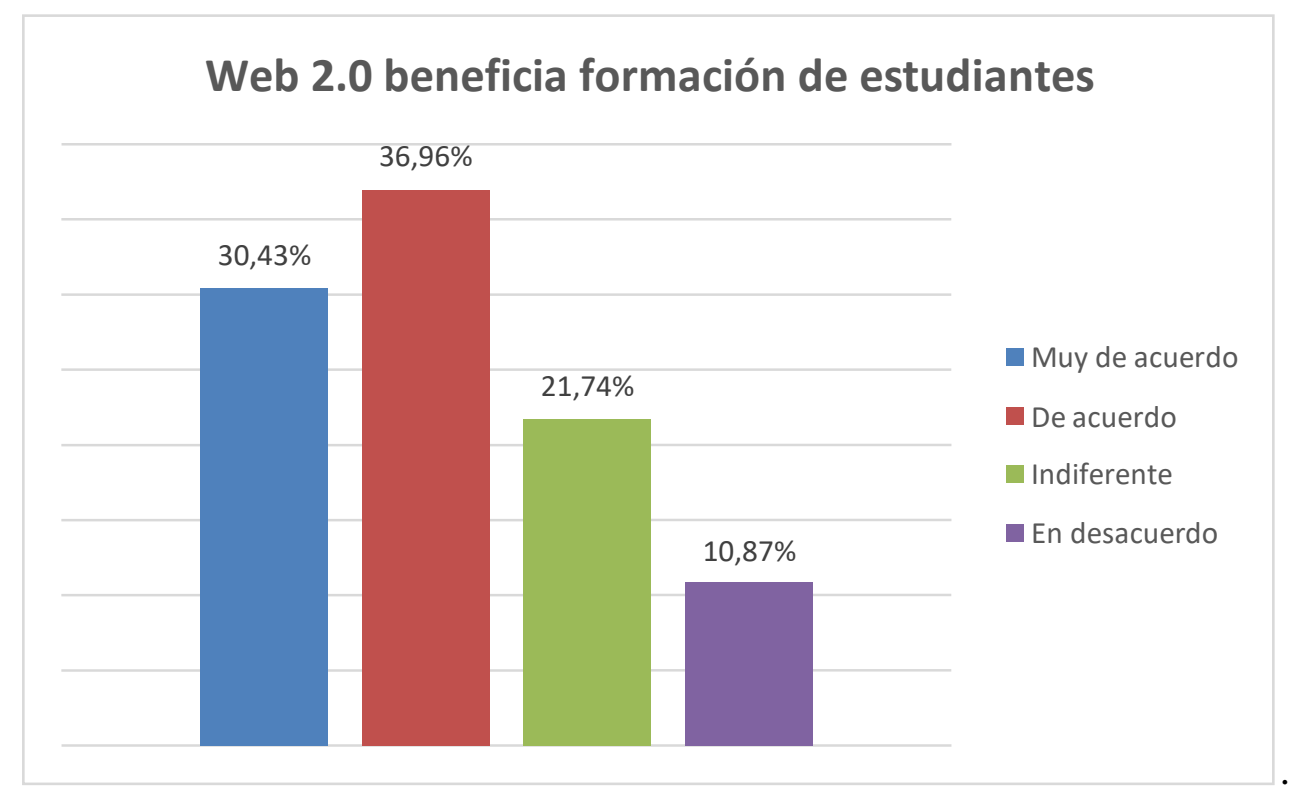

Gráfico 10: Beneficio Web 2.0 en la formación de estudiantes

\section{Análisis}

El 30,43\% de los docentes encuestados están muy de acuerdo que la Web 2.0 beneficia la formación de los estudiantes, el 36,96\% de acuerdo, 21,74\% indiferente y el 10,87\% en desacuerdo.

La mayoría de los docentes está de acuerdo porque aporta a la mejora de la calidad de la educación. 


\begin{tabular}{lcc}
\hline \multicolumn{1}{c}{ Alternativas } & Cantidad & \% \\
\hline Muy de acuerdo & 39 & $84,78 \%$ \\
De acuerdo & 5 & $10,87 \%$ \\
Indiferente & 2 & $4,35 \%$ \\
En desacuerdo & 0 & $0,00 \%$ \\
Total & 46 & $100,00 \%$ \\
\hline
\end{tabular}

Pregunta 11: ¿Cree usted que en el curso de nivelación por carrera de la Universidad Estatal de Milagro se debe implementar un manual sobre el uso educativo de la web 2.0?

Cuadro 21: Implementación de manual sobre el uso web 2.0

Fuente: Encuesta realizada a los docentes del curso de nivelación SNNA-UNEMI

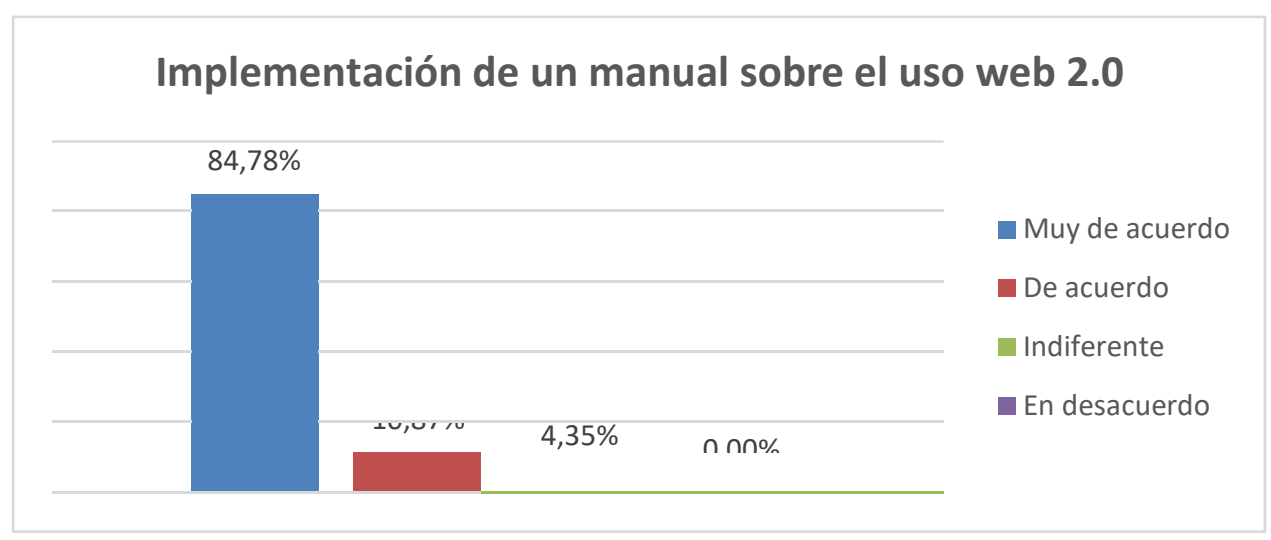

Gráfico 21: Implementación de manual sobre el uso web 2.0

Fuente: Encuesta realizada a los docentes del curso de nivelación SNNA-UNEMI

\section{Análisis}

De los docentes encuestados, el 84,78\% están muy de acuerdo que en el curso de nivelación se debe implementar un manual sobre el uso educativo de la web 2.0, el 10,87\% de acuerdo, el $4,35 \%$ indiferente y el $0 \%$ en desacuerdo.

En los resultados obtenidos se puede apreciar que hay una gran aceptación por parte del docente en innovar sus clases y brindar nuevos entornos de aprendizaje. 


\begin{tabular}{lcc}
\hline \multicolumn{1}{c}{ Alternativas } & Cantidad & \% \\
\hline Muy de acuerdo & 39 & $84,78 \%$ \\
De acuerdo & 7 & $15,22 \%$ \\
Indiferente & 0 & $0,00 \%$ \\
En desacuerdo & 0 & $0,00 \%$ \\
Total & 46 & $100,00 \%$ \\
\hline
\end{tabular}

Pregunta 12: ¿Cree usted que la aplicación de un manual de uso de herramientas de la web 2.0 en el curso de nivelación por carrera de la Universidad Estatal de Milagro aumentará el nivel de comunicación entre docente y estudiante?

Cuadro 32: Aplicación de manual uso de la web 2.0

Fuente: Encuesta realizada a los docentes del curso de nivelación SNNA-UNEMI

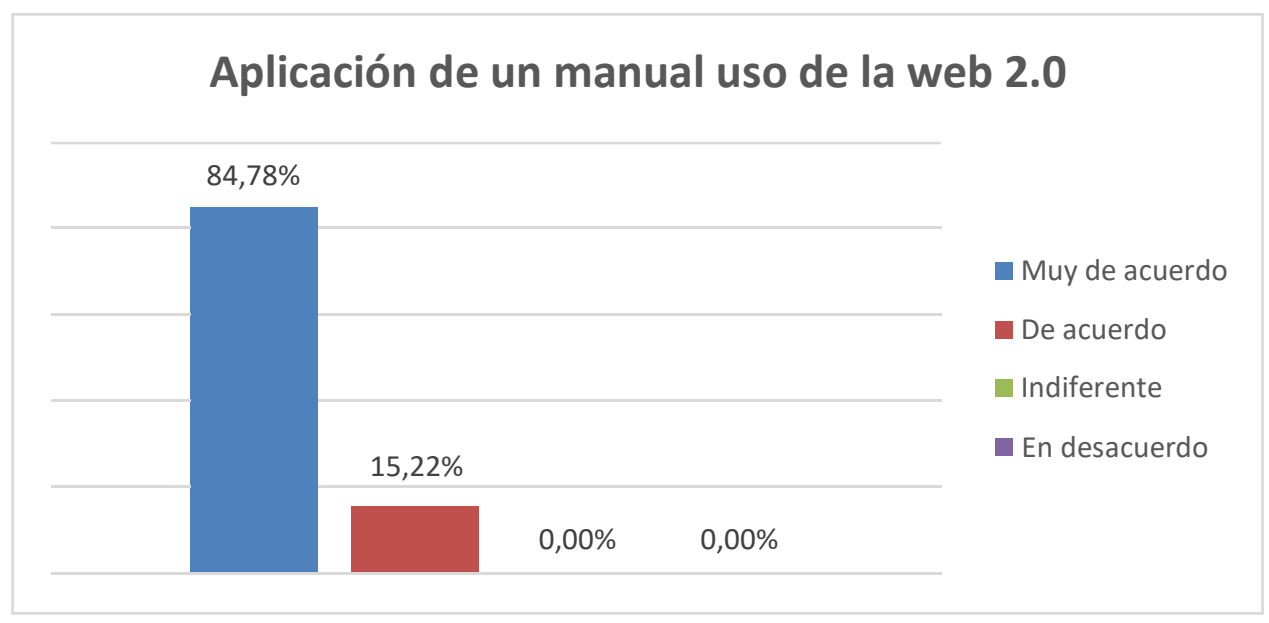

Gráfico 32: Aplicación de manual uso de la web 2.0

Fuente: Encuesta realizada a los docentes del curso de nivelación SNNA-UNEMI

\section{Análisis}

De los docentes encuestados el 84,78\% están muy de acuerdo de la aplicación de un manual de uso de herramientas de la web 2.0 en el curso de nivelación, el 15,22\% de acuerdo, el $0 \%$ indiferente y el $0 \%$ en desacuerdo.

El 100\% de los encuestados están de acuerdo en la aplicación de un manual sobre el uso de las herramientas tecnológicas con el propósito de mejorar el nivel de comunicación entre el estudiante y docente. 


\begin{tabular}{lcc}
\hline \multicolumn{1}{c}{ Alternativas } & Cantidad & $\%$ \\
\hline Muy de acuerdo & 46 & $100,00 \%$ \\
De acuerdo & 0 & $0,00 \%$ \\
Indiferente & 0 & $0,00 \%$ \\
En desacuerdo & 0 & $0,00 \%$ \\
Total & 46 & $100,00 \%$ \\
\hline
\end{tabular}

Pregunta 13: ¿EI Uso de la web 2.0 en su categoría de comunicación como Facebook, Slideshare y Youtube, contribuye positivamente al nivel de sus competencias tecnológicas?

Cuadro 43: Uso de la Web en su categoría de comunicación Fuente: Encuesta realizada a los docentes del curso de nivelación SNNA-UNEMI

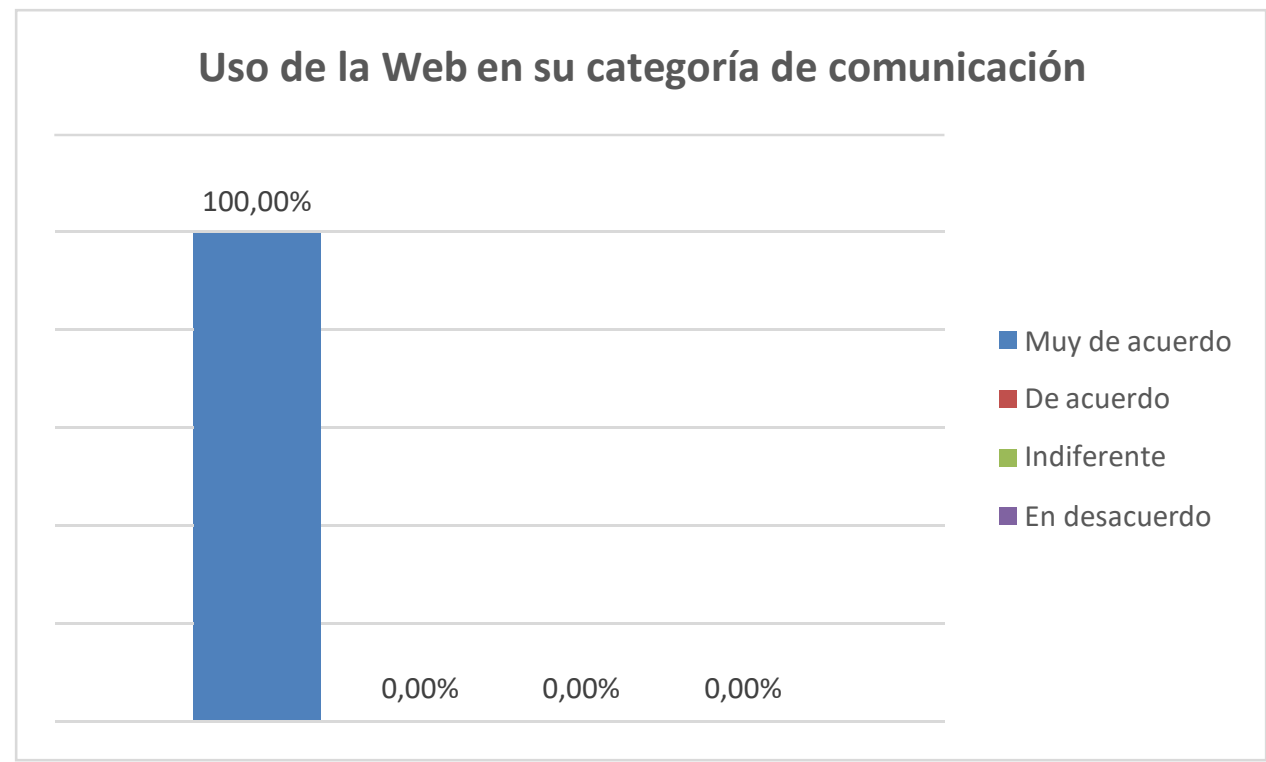

Gráfico 13: Uso de la Web en su categoría de comunicación Fuente: Encuesta realizada a los docentes del curso de nivelación SNNA-UNEMI

\section{Análisis}

El 100\% de los docentes encuestados están muy de acuerdo con esta pregunta e indican que el uso de la web 2.0 en su categoría de comunicación como Facebook, Slideshare y youtube, contribuye positivamente al nivel de competencias tecnológicas de los docentes. 
Renzo R. Padilla-Gómez; Silvia M. Chicaiza-Guayta; María M. Amaguaya-Vargas

\begin{tabular}{lccc}
\hline & Alternativas & Cantidad & $\%$ \\
\hline Mucho & 2 & $4,35 \%$ \\
Poco & 10 & $21,74 \%$ \\
Nada & 34 & $73,91 \%$ \\
Total & 46 & $100,00 \%$ \\
\hline
\end{tabular}

\section{Pregunta 14}

¿Utiliza herramientas tecnológicas para el desarrollo de su clase?

Cuadro 14: Uso de herramientas tecnológicas desarrollo de clase Fuente: Encuesta realizada a los docentes del curso de nivelación SNNA-UNEMI

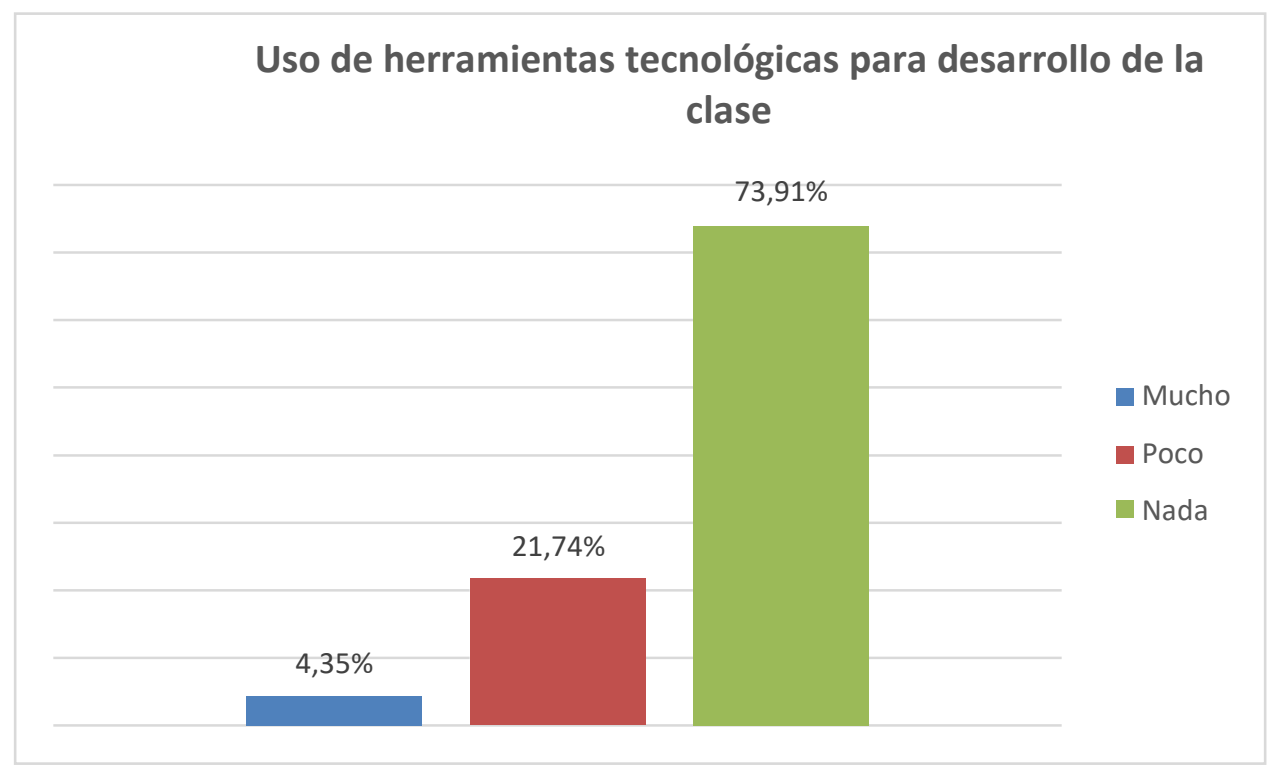

Gráfico 14: Uso de herramientas tecnológicas desarrollo de clase

Fuente: Encuesta realizada a los docentes del curso de nivelación SNNA-UNEMI

\section{Análisis}

De los docentes encuestados el 4,35\% utilizan mucho las herramientas tecnológicas para el desarrollo de su clase, el 21,74\% poco, el 73,91\% nada.

Se puede apreciar que la mayoría de los docentes usan métodos obsoletos para desarrollar sus clases y no hacen uso de herramientas tecnológicas. 


\section{ENCUESTA A ESTUDIANTES}

\begin{tabular}{lcc}
\hline \multicolumn{1}{c}{ Alternativas } & Cantidad & $\%$ \\
\hline Hogar & 167 & $58,80 \%$ \\
Universidad & 284 & $100,00 \%$ \\
Cyber & 3 & $1,06 \%$ \\
Otros & 75 & $26,41 \%$ \\
Total & & \\
\hline
\end{tabular}

\section{Pregunta $1:$ ¿Tiene acceso a internet en?}

Cuadro 55: Acceso a internet

Fuente: Encuesta realizada a los estudiantes del curso de nivelación SNNA-UNEMI

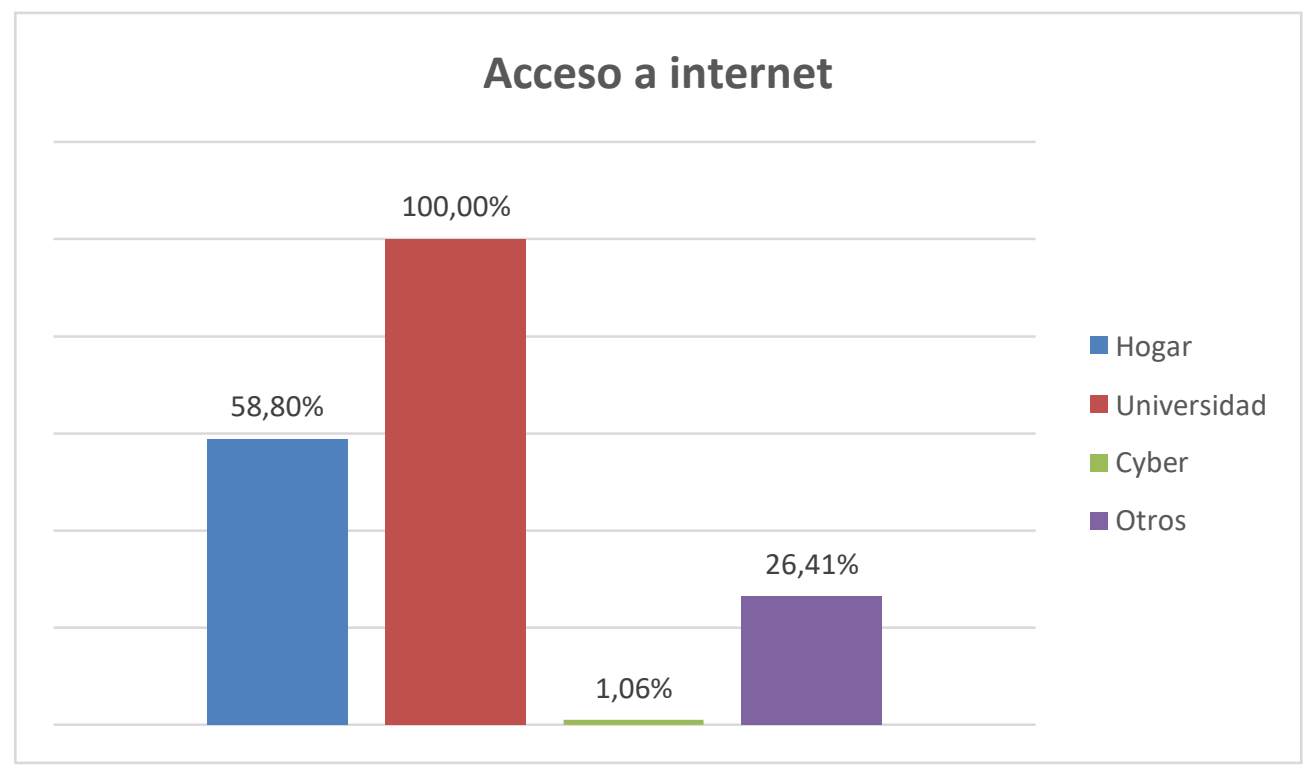

Gráfico 15: Acceso a internet

Fuente: Encuesta realizada a los estudiantes del curso de nivelación SNNA-UNEMI

\section{Análisis}

El 58,80\% de los estudiantes encuestados indican que tienen acceso a internet en el hogar, el $100 \%$ en la universidad, el 1,06\% en el cyber y el 26,41\% otros (celular, parques, mall, entre otros).

En los resultados obtenidos por los estudiantes se puede apreciar que todos tienen acceso a internet en la universidad y la gran mayoría fuera de ésta. 


\begin{tabular}{lcc}
\hline \multicolumn{1}{c}{ Alternativas } & Cantidad & \% \\
\hline Todos los días & 260 & $91,55 \%$ \\
Varios días a la semana & 22 & $7,75 \%$ \\
Una vez a la semana & 2 & $0,70 \%$ \\
Nunca & 0 & $0,00 \%$ \\
Total & 284 & $100,00 \%$ \\
\hline
\end{tabular}

Pregunta $2:$ ¿Con que frecuencia acceso a internet:?

Cuadro 16: Frecuencia acceso a internet

Fuente: Encuesta realizada a los estudiantes del curso de nivelación SNNA-UNEMI

Frecuencia acceso a internet

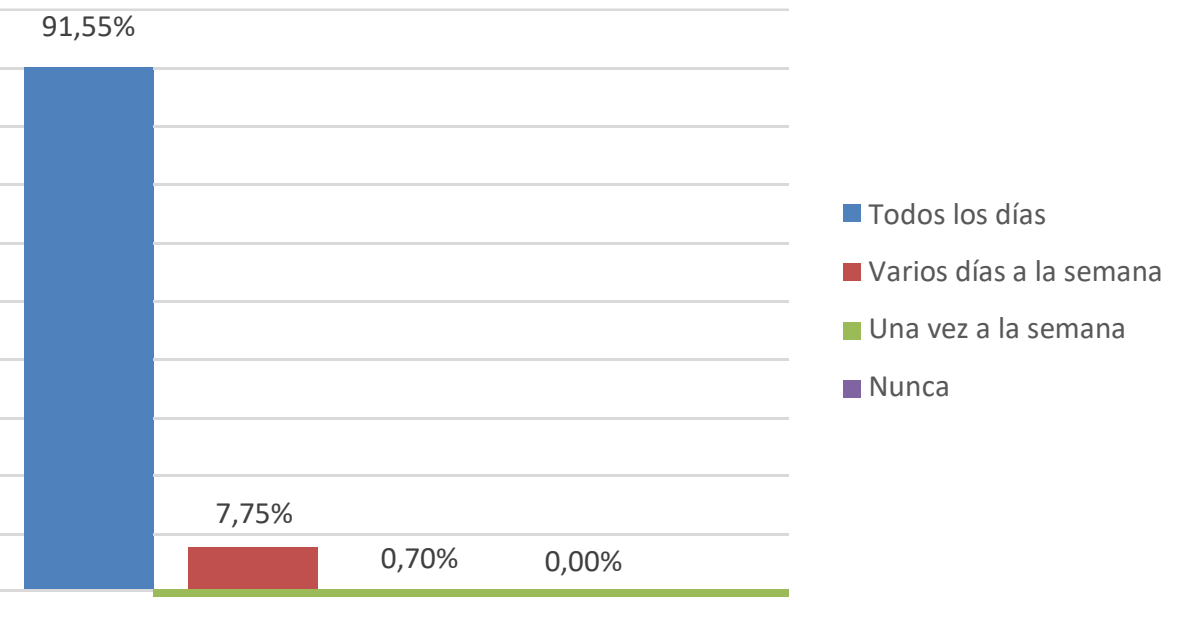

Gráfico 16: Frecuencia acceso a internet

Fuente: Encuesta realizada a los estudiantes del curso de nivelación SNNA-UNEMI Análisis

El 91,55\% de los estudiantes encuestados indican que todos los días accesan a internet, el $7,75 \%$ varios días a la semana, el $0,70 \%$ una vez a la semana, el $0 \%$ nunca.

En los resultados obtenidos por los estudiantes se puede apreciar que todos accesan a internet la mayor parte del tiempo.

\begin{tabular}{lcc}
\hline \multicolumn{1}{c}{ Alternativas } & Cantidad & \% \\
\hline Compartir información & 30 & $10,56 \%$ \\
Comunicación (email,facebook,etc) & 80 & $28,17 \%$ \\
Entretenimiento & 169 & $59,51 \%$ \\
Otros & 5 & $1,76 \%$ \\
Total & 284 & $100,00 \%$ \\
\hline
\end{tabular}

Pregunta 3: ¿Cuál de las siguientes actividades realiza con frecuencia en el internet? 


\section{Cuadro 17: Actividades frecuentes en el internet}

Fuente: Encuesta realizada a los estudiantes del curso de nivelación SNNA-UNEMI

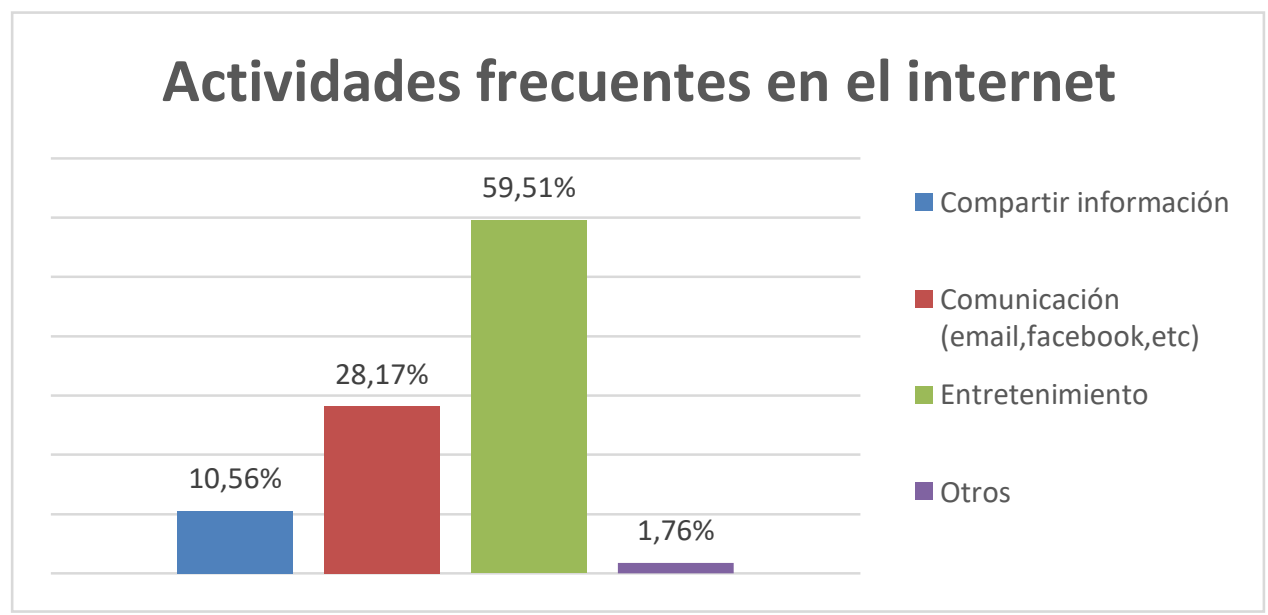

Gráfico 17: Actividades frecuentes en el internet

Fuente: Encuesta realizada a los estudiantes del curso de nivelación SNNA-UNEMI

\section{Análisis}

De los estudiantes encuestados sobre las actividades que realiza con frecuencia en el internet el 10,56\% comparte información, el 28,17\% tiene comunicación atreves de email, Facebook, el $59,51 \%$ por entretenimiento, el $1,76 \%$ otros.

En los resultados obtenidos por los estudiantes se puede apreciar que la tendencia es la comunicación y entretenimiento, es decir que lo usan para diversión.

\begin{tabular}{lcc}
\hline \multicolumn{1}{c}{ Alternativas } & Cantidad & \% \\
\hline Facebook & 6 & $2,11 \%$ \\
Google Drive & 19 & $6,69 \%$ \\
Slideshare & 6 & $2,11 \%$ \\
Youtube & 81 & $28,52 \%$ \\
Ninguna & 172 & $60,56 \%$ \\
Total & 284 & $100,00 \%$ \\
\hline
\end{tabular}

Pregunta 4: ¿De las siguientes herramientas cual utiliza el docente en el proceso de enseñanza aprendizaje?

Cuadro 18: Herramientas que utiliza el docente

Fuente: Encuesta realizada a los estudiantes del curso de nivelación SNNA-UNEMI 


\section{Herramientas que utiliza el docente}

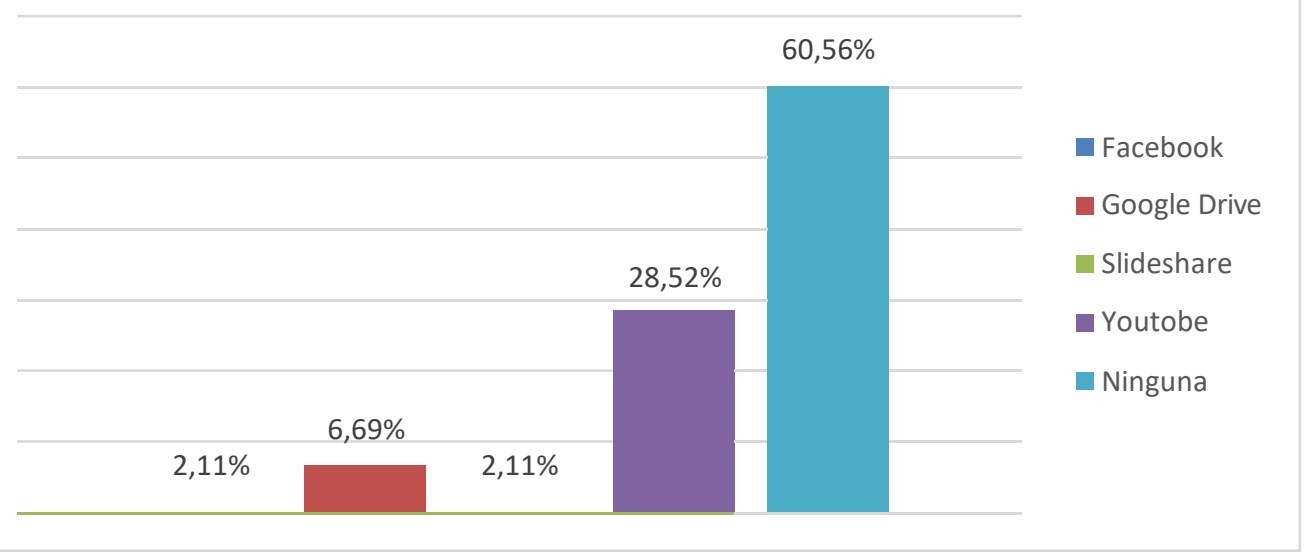

Gráfico 18: Herramientas que utiliza el docente

Fuente: Encuesta realizada a los estudiantes del curso de nivelación SNNA-UNEMI Análisis

De los estudiantes encuestados sobre las herramientas que utiliza el docente en el proceso de enseñanza aprendizaje, el 2,11\% usa Facebook, el 6,69\% google drive, el 2,11\% Slideshare, el $28,52 \%$ Youtube, $60,56 \%$ ninguna.

En los resultados obtenidos por los estudiantes se puede apreciar que la mayoría usa Facebook y Youtube pero no para fines educativos sino para diversión.

\begin{tabular}{lcc}
\hline \multicolumn{1}{c}{ Alternativas } & Cantidad & \% \\
\hline Facebook & 6 & $2,11 \%$ \\
Google Drive & 19 & $6,69 \%$ \\
Slideshare & 6 & $2,11 \%$ \\
Youtube & 81 & $28,52 \%$ \\
Otros & 172 & $60,56 \%$ \\
Total & 284 & $100,00 \%$ \\
\hline
\end{tabular}

Pregunta 5: ¿Utiliza usted alguna herramienta tecnológica para el desarrollo de sus actividades escolares?

Cuadro 19: Frecuencia de utilización de herramientas tecnológicas para el desarrollo de actividades escolares

Fuente: Encuesta realizada a los estudiantes del curso de nivelación SNNA-UNEMI 


\section{Herramientas tecnológicas para el desarrollo de actividades}

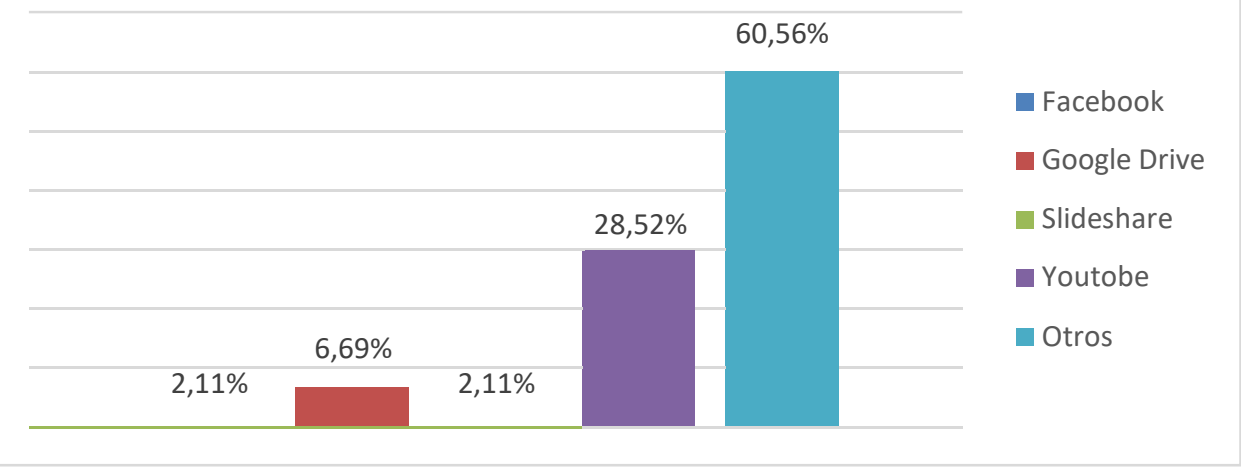

\section{Gráfico 19: Frecuencia de utilización de herramientas tecnológicas para el desarrollo de} actividades escolares Análisis

Fuente: Encuesta realizada a los estudiantes del curso de nivelación SNNA-UNEMI

De los estudiantes encuestados mencionan que de las herramientas que utiliza el docente en el proceso de enseñanza aprendizaje, el 2,11\% es el Facebook, el 6,69\% Google Drive, el 2,11\% Slideshare, el 28,52\% Youtube, el 60,56\% otros.

En los resultados obtenidos por los estudiantes, se puede apreciar que hace uso de otra herramienta para el desarrollo de actividades escolares.

\begin{tabular}{lcc}
\hline \multicolumn{1}{c}{ Alternativas } & Cantidad & \% \\
\hline Educación & 72 & $25,35 \%$ \\
Conocer Personas & 113 & $39,79 \%$ \\
Entretenimiento & 90 & $31,69 \%$ \\
No utiliza & 9 & $3,17 \%$ \\
Total & 284 & $100,00 \%$ \\
\hline
\end{tabular}

Pregunta 6: ¿En qué ámbitos ha utilizado las herramientas Web 2.0 tales como facebook, Google Drive, Slideshare, Youtube?

Cuadro 20: Ámbitos en que utiliza la web 2.0

Fuente: Encuesta realizada a los estudiantes del curso de nivelación SNNA-UNEMI 


\section{Ámbitos en que utiliza la web 2.0}

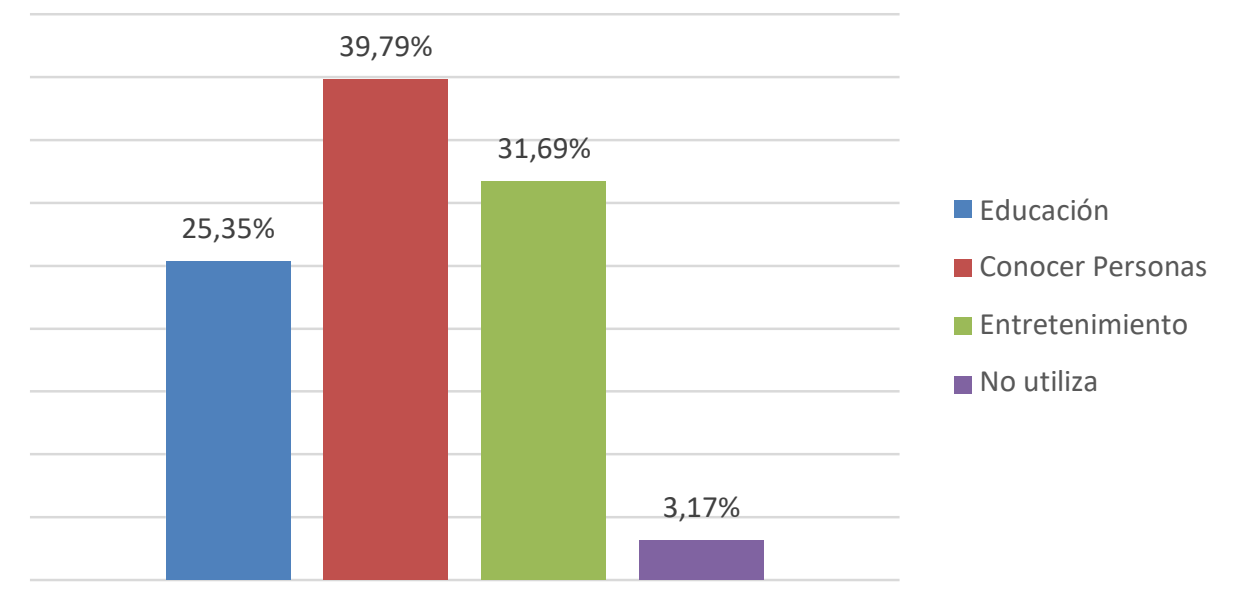

Gráfico 20: Ámbitos en que utiliza la web 2.0

Fuente: Encuesta realizada a los estudiantes del curso de nivelación SNNA-UNEMI

\section{Análisis}

De los estudiantes encuestados, sobre los ámbitos que utiliza las herramientas Web 2.0, el 25,35\% en educación, el 39,79\% Conocer personas, el 31,69\% entretenimiento, 3,17\% no utiliza.

En los resultados obtenidos por los estudiantes se puede apreciar que una menor parte lo usan para el ámbito educativo, más es usado para conocer personas y entretenimiento.

\begin{tabular}{lcc}
\hline \multicolumn{1}{c}{ Alternativas } & Cantidad & $\%$ \\
\hline Muy de acuerdo & 180 & $63,38 \%$ \\
De acuerdo & 103 & $36,27 \%$ \\
Indiferente & 1 & $0,35 \%$ \\
En desacuerdo & 0 & $0,00 \%$ \\
Total & 284 & $100,00 \%$ \\
\hline
\end{tabular}

Pregunta 7: ¿Usted considera que el envío y recepción de tareas se lo realice por medio de herramientas tecnológicas en la web?

Cuadro 21: Envío y recepción de tareas por medio de herramientas tecnológicas Fuente: Encuesta realizada a los estudiantes del curso de nivelación SNNA-UNEMI 


\section{Envío y recepción de tareas por medio de herramentas tecnológicas}

$63,38 \%$

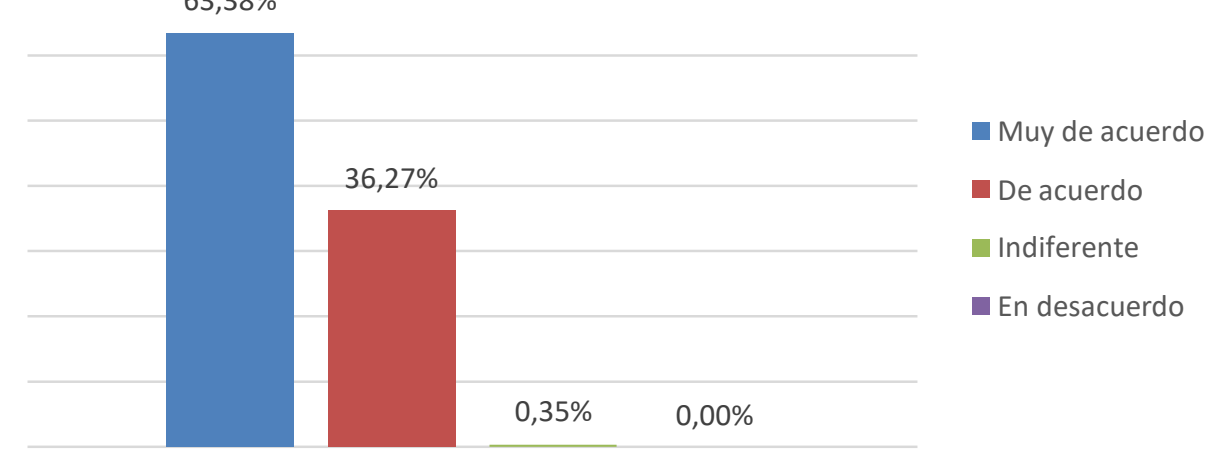

Gráfico 21: Envío y recepción de tareas por medio de herramientas tecnológicas Fuente: Encuesta realizada a los estudiantes del curso de nivelación SNNA-UNEMI Análisis

De los estudiantes encuestados el 63,38\% indican que están muy de acuerdo que el envío y recepción de tareas se lo realice por medio de herramientas tecnológicas en la web, el 36,27\% de acuerdo, el 0,35\% indiferente, el $0 \%$ en desacuerdo.

En los resultados obtenidos por los estudiantes se puede apreciar que hay una gran aceptación que se utilice herramientas tecnológicas para presentación de tareas

\begin{tabular}{lcc}
\hline \multicolumn{1}{c}{ Alternativas } & Cantidad & \% \\
\hline Muy de acuerdo & 4 & $1,41 \%$ \\
De acuerdo & 20 & $7,04 \%$ \\
Indiferente & 140 & $49,30 \%$ \\
En desacuerdo & 120 & $42,25 \%$ \\
Total & 284 & $100,00 \%$ \\
\hline
\end{tabular}

Pregunta 8: ¿Usted sabía que Facebook, Google Drive, Slideshare, Youtube son herramientas web 2.0?

Cuadro 22: Conocimiento sobre herramientas web 2.0

Fuente: Encuesta realizada a los estudiantes del curso de nivelación SNNA-UNEMI 


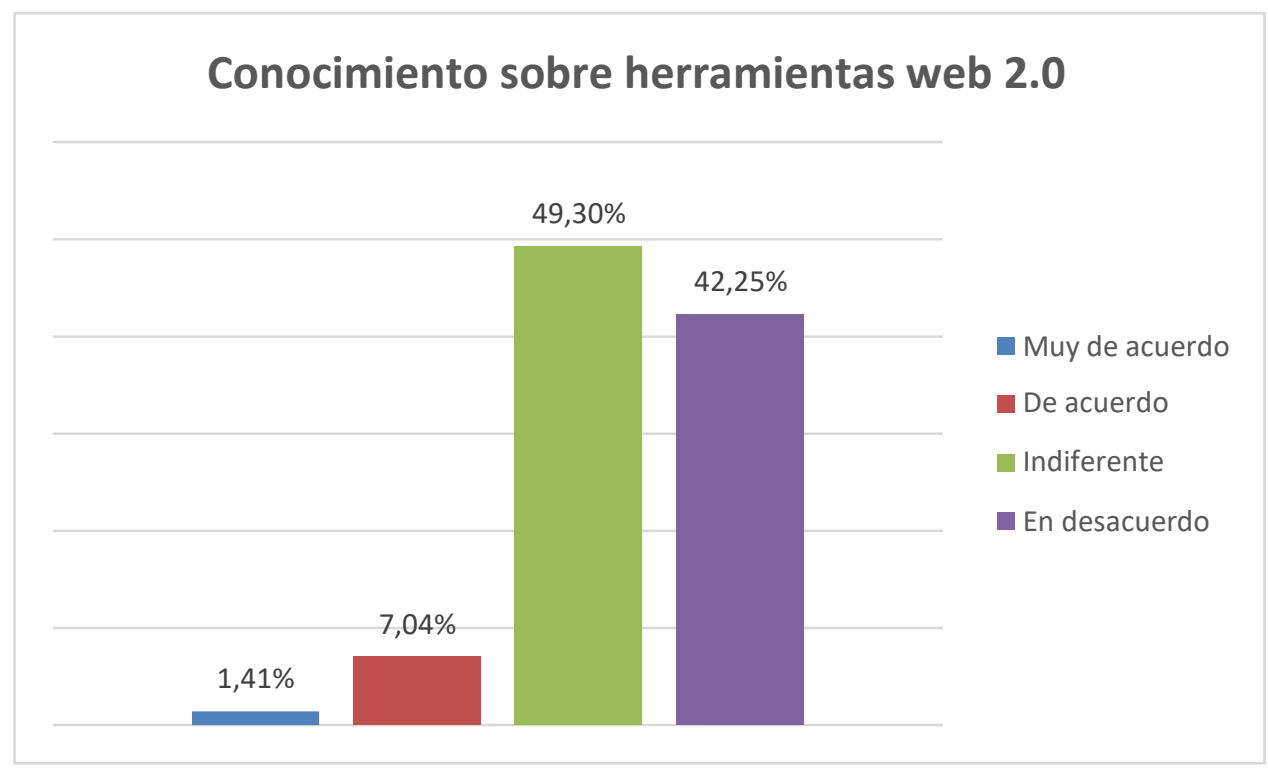

Gráfico 22: Conocimiento sobre herramientas web 2.0

Fuente: Encuesta realizada a los estudiantes del curso de nivelación SNNA-UNEMI Análisis

De los estudiantes encuestados, el 1,41\% está muy de acuerdo lo que son herramientas web, el 7,04\% de acuerdo, el 49,30\% indiferente, el 42,25\% en desacuerdo.

La mayoría de los encuestados desconoce que esas herramientas que pueden ser de gran ayuda en el área educativa son parte de la web 2.0.

\begin{tabular}{lcc}
\hline \multicolumn{1}{c}{ Alternativas } & Cantidad & \% \\
\hline Muy de acuerdo & 150 & $52,82 \%$ \\
De acuerdo & 133 & $46,83 \%$ \\
Indiferente & 1 & $0,35 \%$ \\
En desacuerdo & 0 & $0,00 \%$ \\
Total & 284 & $100,00 \%$ \\
\hline
\end{tabular}

Pregunta 9: ¿Le gustaría usar algunas de las herramientas web 2.0 como medios de comunicación entre sus compañeros de estudio y maestros?

Cuadro 23: Herramientas web 2.0 como medios de comunicación

Fuente: Encuesta realizada a los estudiantes del curso de nivelación SNNA-UNEMI 


\section{Herramientas web 2.0 como medios de comunicación}

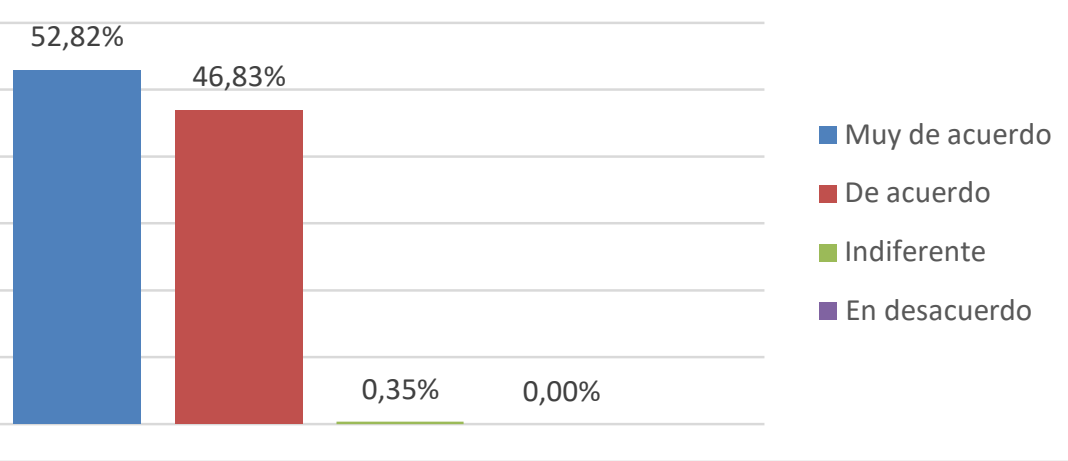

Gráfico 23: Herramientas web 2.0 como medios de comunicación Fuente: Encuesta realizada a los estudiantes del curso de nivelación SNNA-UNEMI

\section{Análisis}

De los estudiantes encuestados, el 52,82\% está muy de acuerdo usar algunas de las herramientas web 2.0 como medios de comunicación en el aula de clases, el 46,83\% de acuerdo, el $0,35 \%$ indiferente, el $0 \%$ en desacuerdo.

Existe bastante aceptación por parte de los estudiantes que se use herramientas de la web 2.0 como una forma de interactuar entre maestros y compañeros.

\begin{tabular}{lcc}
\hline \multicolumn{1}{c}{ Alternativas } & Cantidad & \% \\
\hline Muy de acuerdo & 159 & $55,99 \%$ \\
De acuerdo & 124 & $43,66 \%$ \\
Indiferente & 1 & $0,35 \%$ \\
En desacuerdo & 0 & $0,00 \%$ \\
Total & 284 & $100,00 \%$ \\
\hline
\end{tabular}

Pregunta 10: ¿Le gustaría tener un espacio online en un tiempo determinado donde le pueda usted preguntar al docente sobre alguna inquietud de la clase?

Cuadro 24: Espacio online para preguntar al docente

Fuente: Encuesta realizada a los estudiantes del curso de nivelación SNNA-UNEMI 


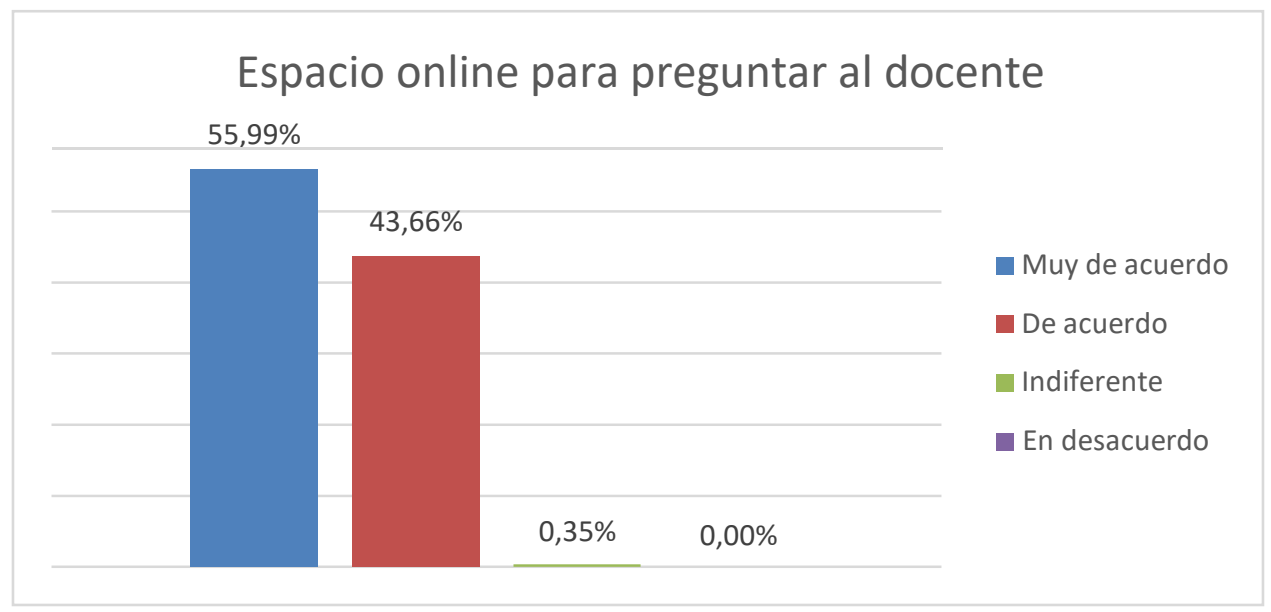

Gráfico 24: Espacio online para preguntar al docente

Fuente: Encuesta realizada a los estudiantes del curso de nivelación SNNA-UNEMI

\section{Análisis}

De los estudiantes encuestados el 55,99\% está muy de acuerdo tener un espacio online donde pueda interactuar con el docente sobre alguna inquietud de la clase, el 43,66\% de acuerdo, el 0,35\% indiferente, $0 \%$ en desacuerdo.

La mayoría de estudiantes están de acuerdo con contar con este tipo de asesoría porque muchas veces tienen que esperar hasta las próximas clases para despejar dudas, ocasionando retrasos en el trabajo.

\begin{tabular}{lcc}
\hline \multicolumn{1}{c}{ Alternativas } & Cantidad & \% \\
\hline Muy de acuerdo & 201 & $70,77 \%$ \\
De acuerdo & 82 & $28,87 \%$ \\
Indiferente & 1 & $0,35 \%$ \\
En desacuerdo & 0 & $0,00 \%$ \\
Total & 284 & $100,00 \%$ \\
\hline
\end{tabular}

Pregunta 11: ¿Está de acuerdo que el docente le deba impulsar a la enseñanza basada en la construcción del conocimiento mediante el uso de las herramientas web 2.0?

Cuadro 25: Impulsar la enseñanza mediante el uso de la web 2.0 Fuente: Encuesta realizada a los estudiantes del curso de nivelación SNNA-UNEMI 


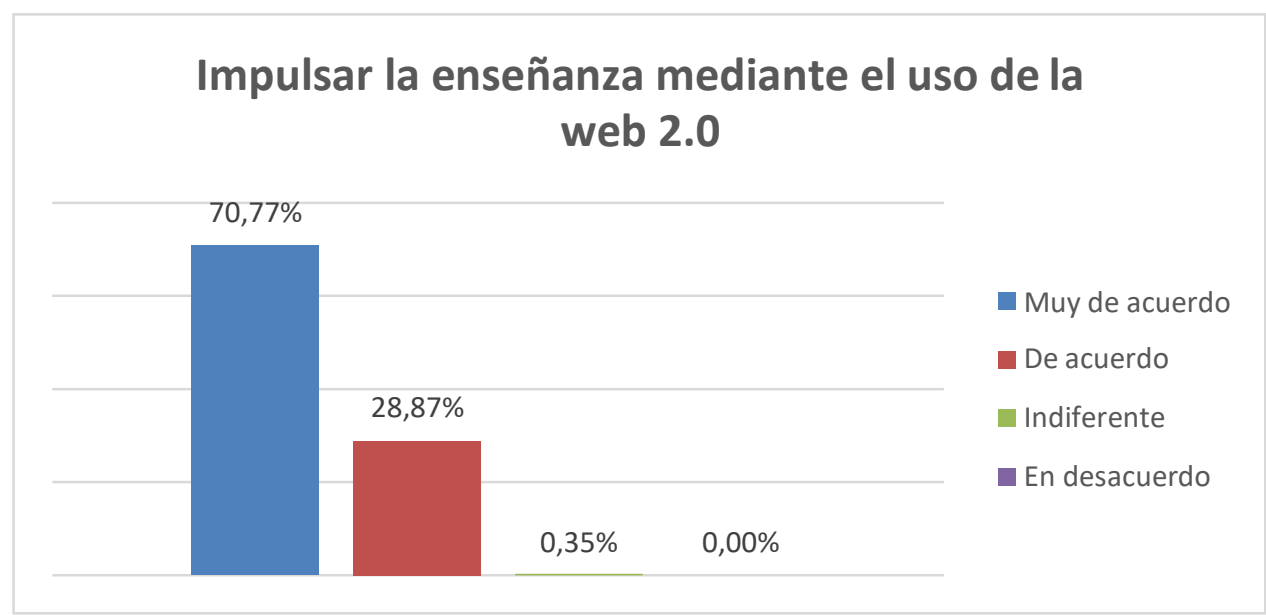

Gráfico 25: Impulsar la enseñanza mediante el uso de la web 2.0

Fuente: Encuesta realizada a los estudiantes del curso de nivelación SNNA-UNEMI

\section{Análisis}

De los estudiantes encuestados, el 70,77\% están muy de acuerdo que los docentes impulsen a la enseñanza basada en la construcción del conocimiento mediante el uso de las herramientas web 2.0, el $28,87 \%$ de acuerdo, el 0,35\% indiferente y el $0 \%$ en desacuerdo.

Existe una gran aceptación por parte del estudiante, por cuanto se pretende dejar atrás la enseñanza tradicional y hacer un cambio donde el estudiante sea el creador de su propio conocimiento con la guía del docente.

\begin{tabular}{lcc}
\hline \multicolumn{1}{c}{ Alternativas } & Cantidad & $\%$ \\
\hline Muy de acuerdo & 214 & $75,35 \%$ \\
De acuerdo & 69 & $24,30 \%$ \\
Indiferente & 1 & $0,35 \%$ \\
En desacuerdo & 0 & $0,00 \%$ \\
Total & 284 & $100,00 \%$ \\
\hline
\end{tabular}

Pregunta 12: ¿Cree usted que el desconocimiento de los docentes sobre el manejo de las herramientas tecnológicas no le permite mejorar el proceso de enseñanza aprendizaje? Cuadro 26: Desconocimiento sobre el manejo de herramientas tecnológicas Fuente: Encuesta realizada a los estudiantes del curso de nivelación SNNA-UNEMI 


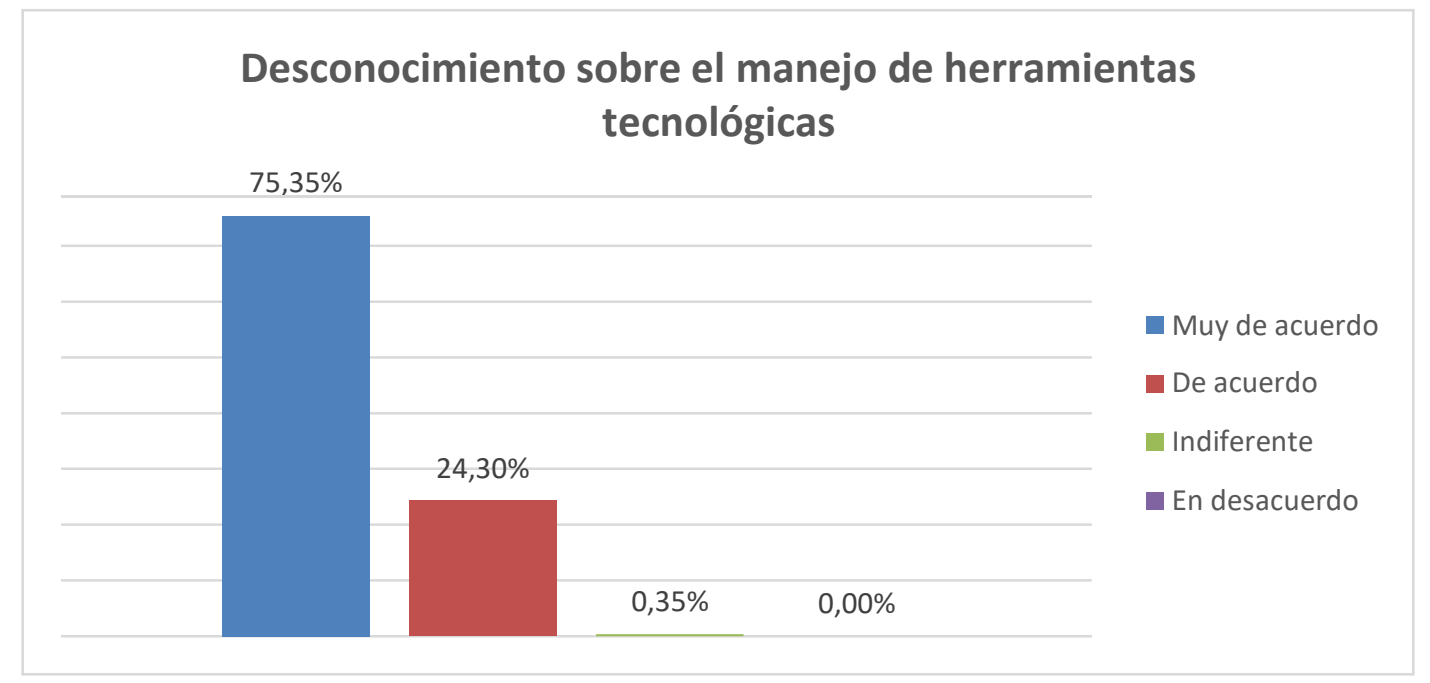

Gráfico 26: Desconocimiento sobre el manejo de herramientas tecnológicas Fuente: Encuesta realizada a los estudiantes del curso de nivelación SNNA-UNEMI

\section{Análisis}

De los estudiantes encuestados, el 75,35\% están muy de acuerdo que el desconocimiento de los docentes sobre el manejo de las herramientas tecnológicas no le permite mejorar el proceso de enseñanza aprendizaje, el 24,30\% de acuerdo, el 0,35\% indiferente, $0 \%$ en desacuerdo.

Los estudiantes están de acuerdo que muchas veces por el poco conocimiento del docente en herramientas tecnológicas no le permite mejorar la educación ni desarrollar clases más interactivas.

\begin{tabular}{lcc}
\hline \multicolumn{1}{c}{ Alternativas } & Cantidad & $\%$ \\
\hline Muy de acuerdo & 284 & $100,00 \%$ \\
De acuerdo & 0 & $0,00 \%$ \\
Indiferente & 0 & $0,00 \%$ \\
En desacuerdo & 0 & $0,00 \%$ \\
Total & 284 & $100,00 \%$ \\
\hline
\end{tabular}

Pregunta 13: ¿EI Uso de la web 2.0 en su categoría de comunicación como Facebook, Slideshare y youtube, contribuye positivamente al nivel de competencias tecnológicas de los docentes?

Cuadro 27: Uso de la Web 2.0 en su categoría de comunicación Fuente: Encuesta realizada a los estudiantes del curso de nivelación SNNA-UNEMI 


\section{Uso de la Web 2.0 en su categoría de comunicación}

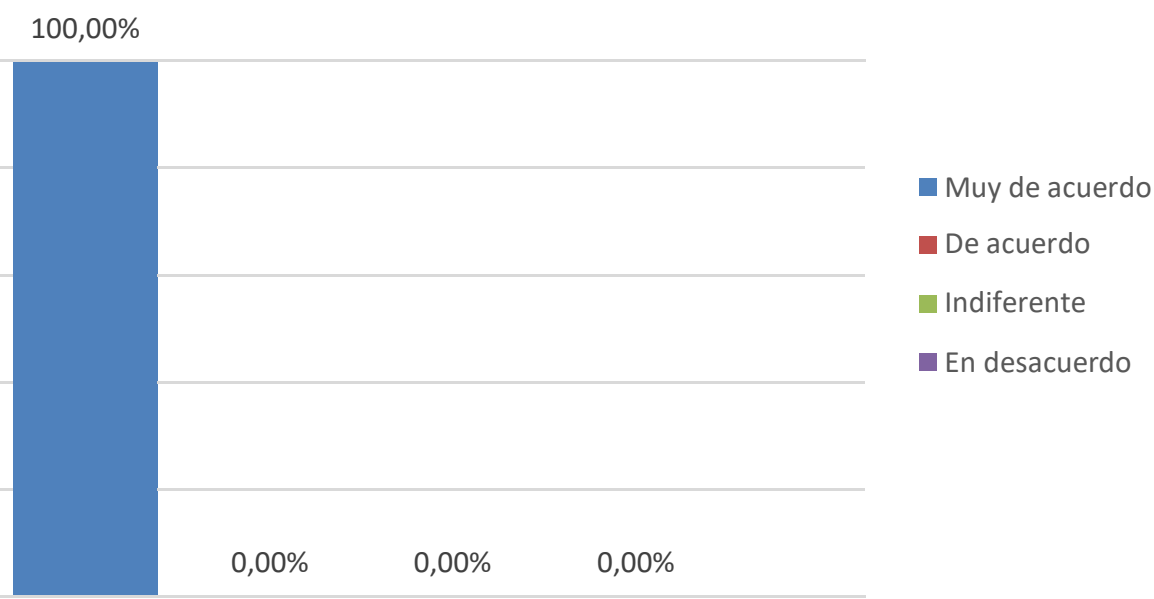

Gráfico 27: Uso de la Web 2.0 en su categoría de comunicación Análisis

Fuente: Encuesta realizada a los estudiantes del curso de nivelación SNNA-UNEMI

El 100\% de los estudiantes encuestados están muy de acuerdo que el uso de la web 2.0 en su categoría de comunicación como Facebook, Slideshare y youtube, contribuye positivamente al nivel de competencias tecnológicas de los docentes.

\begin{tabular}{lcc}
\hline \multicolumn{1}{c}{ Alternativas } & Cantidad & \% \\
\hline Muy de acuerdo & 168 & $59,15 \%$ \\
De acuerdo & 115 & $40,49 \%$ \\
Indiferente & 1 & $0,35 \%$ \\
En desacuerdo & 0 & $0,00 \%$ \\
Total & 284 & $100,00 \%$ \\
\hline
\end{tabular}

Pregunta 14: ¿Le gustaría que los docentes tengan conocimiento de herramientas tecnológicas y éstas las apliquen en el proceso de enseñanza aprendizaje?

Cuadro 28: Docentes con conocimiento de herramientas tecnológicas

Fuente: Encuesta realizada a los estudiantes del curso de nivelación SNNA-UNEMI 


\section{Docentes con conocimiento de herramientas tecnológicas}

$59,15 \%$
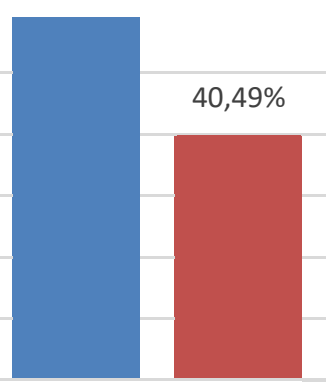

$0,35 \%$

$0,00 \%$
- Muy de acuerdo

- De acuerdo

Indiferente

- En desacuerdo

Gráfico 28: Docentes con conocimiento de herramientas tecnológicas Fuente: Encuesta realizada a los estudiantes del curso de nivelación SNNA-UNEMI

\section{Análisis}

De los estudiantes encuestados, el 59,15\% están muy de acuerdo que los docentes tengan conocimiento de herramientas tecnológicas y que las apliquen en el proceso de enseñanza aprendizaje, el 40,49\% de acuerdo, el 0,35\% indiferente, $0 \%$ en desacuerdo.

Casi la totalidad de los estudiantes están de acuerdo que el docente debe de cambiar la metodología de enseñanza y aplicar estrategias innovadoras.

De la información obtenida por medio de la encuesta a los docentes y estudiantes del curso de nivelación y admisión de la Universidad Estatal de Milagro, se determina la factibilidad del diseño de un manual académico tecnológico para los docentes, la misma que beneficia en el proceso de enseñanza aprendizaje logrando la retroalimentación de información entre educadores y educando.

De las preguntas se les realizó a los estudiantes y docentes tenemos el siguiente análisis:

- El gran número de docentes y estudiantes accesan a internet a realizar actividades de entretenimiento y comunicación entre familiares y amigos utilizando Facebook y youtube.

- La única herramienta que utiliza el docente para comunicación con los estudiantes es el aula virtual implementado por la Universidad Estatal de Milagro. 
- En los resultados obtenidos por los docentes y estudiantes se puede apreciar que hay una gran aceptación en el uso de las herramientas web 2.0, para mejorar su perfil tecnológico y así estar a la vanguardia de la tecnología.

- En los resultados obtenidos se puede dar cuenta que hay una gran aprobación por parte del docente en innovar sus clases y brindar nuevos entornos de aprendizaje.

- Existe una gran aceptación por parte del estudiante, por cuanto se pretende dejar atrás la enseñanza tradicional y hacer un cambio donde el estudiante sea el creador de su propio conocimiento con la guía del docente.

- Los estudiantes están de acuerdo que muchas veces por el poco conocimiento del docente en herramientas tecnológicas no le permite mejorar la educación ni desarrollar clases más interactivas.

- Casi la totalidad de los estudiantes están de acuerdo que el docente debe de cambiar la metodología de enseñanza y aplicar estrategias innovadoras.

De las entrevistas realizadas a los coordinadores se puede concluir que hay una aceptación del $100 \%$ con la implementación de un manual académico tecnológico para los docentes, ya que les permite a los jóvenes aprendan en un entorno más colaborativo

\section{Conclusiones}

- La formación del docente debe de ir encaminada en cuanto al manejo de aplicaciones tecnológicas que provienen de las herramientas web 2.0, como la capacitación pedagógica y didáctica necesaria para utilizar e integrar estos recursos en los planes analíticos y así mejorar el proceso de enseñanza aprendizaje.

- La incorporación de las herramientas de la web 2.0 en el ámbito de la educación universitaria abre muchas posibilidades ante muchos cambios que exige la educación, enfatizando las nuevas formas de aprendizaje, comunicación e investigación.

- Es importante que el docente ponga en claro las reglas con los estudiantes para realizar las actividades usando las herramientas web 2.0 para evitar que se distraigan con actividades no escolares. 


\section{Bibliografía.}

UNESCO. (08 de 01 de 2008). Recuperado el 22 de 07 de 2015, de Organización de las Naciones Unidas para la Educación, Ciencia y Cultura: http://portal.unesco.org/es/ev.phpURL_ID=41553\&URL_DO=DO_TOPIC\&URL_SECTION=201.html

Acuña, B. P. (2011). Métodos científicos de observación en Educación. Madrid: Liber Factory.

Alvarez, T. (2001). La Escuela en la vida. Habana: Pueblo y Educación.

Arias, M. (2003). El espacio Europeo de Educación Superior: Una oportunidad de desarrollo multidisciplinar a través del aprendizaje y la tecnolía.

Bartolomé, A. (2008). La Web 2.0 paradigmas y nuevos aprendizajes.

Cabreiro, B. (2007). Las nuevas tecnologías como instrumentos didácticos. Madrid: Tecnología Educativa.

Cobo, C., \& Pardo, H. (2007). Planeta Web 2.0. Inteligencia colectiva o medios fast food. Universidad de Vic.

Cornejo Méndez, E. V. (Octubre de 2013). El desarrollo de las competencias cognitivas básicas a través del uso del facebook como herramienta mediadora del proceso de apendizaje. 24. Colombia.

De Haro, J. J. (2009). Algunas experiencias de innovación educativa. Arbor.

Díaz, F. A. (26 de 03 de 2012). TICS. Obtenido de http://www.slideshare.net/faber1226/las-ticss

EDUCAUSE. (06 de 11 de 2012). Educause annual conference 2012. Recuperado el 22 de 07 de 2015, de http://www.educause.edu/annual-conference/2012

Esteve, F. (2009). Bolonia y las TIC: de la docencia 1.0 al aprendizaje 2.0. La Cuestión Universitaria. N. ${ }^{\circ} 5$.

Francisco, A., Traver, J., \& Marco, F. (2011). Promover el compromiso cívico en la educación superior. XII Congreso Internacional de Teoría de la Educación. Barcelona.

Garcia Aretio, L., Ruiz, M., \& García, M. (2009). Claves de la Educación. España: Narcea.

García Sans, A. (2008). Las redes sociales como herramientas para el aprendizaje colaborativo: una experiencia con Facebook. Actas del XIII Congreso Internacional en Tecnologías para la Educación. Madrid: UNED.

Guereca, C., Mendoza, C., Bogdanski, T., Ramirez, D., \& Santana, P. (2013). Valores para la vida. UNID.

Hernández Requena, S. (2008). El modelo constructivista con las nuevas tecnologías: aplicado en el proceso de aprendizaje. Revista de Universidad y Sociedad del Conocimiento, 26-35.

Marcelo, C., \& Vaillant, D. (2009). Desarrollo Profesional Docente ¿Cómo se aprende a enseñar? Málaga: Narcea.

Marqués, P. (2007). La Web 2.0 y sus Aplicaciones Didácticas. Santiago de Chile.

Micolini, A. (2006). Competencias para un estudio eficaz. Argentina: Brujas.

Moral, D. (2007). Una presentación multimedia de un proyecto conjunto para un título en educación. Canada: Quebec. 
Piscitelli, A. A. (2010). El proyecto Facebook y la Posuniversidad. Sistemas Operativos Sociales y Entornos Abiertos de Aprendizaje. Madrid: Ariel.

Quintana. (2000). Competencias en tecnologías de la información del profesorado. Revista Inter Universitaria de tecnología Educativa, 166-174.

Reyero, Garcia, Hernández, \& Ovide. (2011). Autonomía y responsbilidad en el contexto de la sociedad de las tecnologías de la información y la comunicación. España.

Secretaria Nacional de Educación Superior, C. t. (2013). SENESCYT. Recuperado el 23 de 07 de 2015, de MODELO DE GESTIÓN DEL SNNA: www.educacionsuperior.gob.ec/wp-content/.../PROYECTOSNNA.pdf 